Draft Version OCtOBER 6, 2018

Preprint typeset using $\mathrm{IATE}_{\mathrm{E}} \mathrm{X}$ style emulateapj v. 11/26/04

\title{
THE ACS VIRGO CLUSTER SURVEY. XIV. ANALYSIS OF COLOR-MAGNITUDE RELATIONS IN GLOBULAR CLUSTER SYSTEMS ${ }^{1}$
}

\author{
Steffen Mieske ${ }^{2}$, Andrés Jordán ${ }^{2,3}$, Patrick Côté ${ }^{4}$, Markus Kissler-Patig $^{2}$, Eric W. Peng ${ }^{4}$, Laura Ferrarese $^{4}$, \\ John P. Blakeslee ${ }^{5,6}$, Simona Mei ${ }^{5}$, David Merritt ${ }^{7}$, John L. Tonry ${ }^{8}$, \& Michael J. West ${ }^{9}$ \\ Draft version October 6, 2018
}

\begin{abstract}
We examine the correlation between globular cluster (GC) color and magnitude using HST/ACS imaging for a sample of 79 early-type galaxies $\left(-21.7<M_{B}<-15.2 \mathrm{mag}\right)$ with accurate surfacebrightness fluctuation distances from the ACS Virgo Cluster Survey. Using the KMM mixture modeling algorithm, we find a highly significant correlation, $\gamma_{z} \equiv \frac{d(g-z)}{d z}=-0.037 \pm 0.004$, between color and magnitude for the subpopulation of blue GCs in the co-added GC color-magnitude diagram of the three brightest Virgo cluster galaxies (M49, M87 and M60). The sense of the correlation is such that brighter GCs are redder than their fainter counterparts. For the single GC systems of M87 and M60, we find similar correlations; M49 does not appear to show a significant trend. There is no correlation between $(g-z)$ and $M_{z}$ for GCs belonging to the red subpopulation. The correlation $\gamma_{g} \equiv \frac{d(g-z)}{d g}$ for the blue subpopulation is much weaker than $\gamma_{z}$. Using Monte Carlo simulations, we attribute this finding to the fact that the blue subpopulation in $M_{g}$ extends to higher luminosities than does the red subpopulation, which biases the KMM fit results. The highly significant correlation between color and $M_{z}$, however, is a real effect: this conclusion is supported by biweight fits to the same color distributions. We identify two environmental dependencies which influence the derived color-magnitude relation: (1) the slope of the color-magnitude relation decreases in significance with decreasing galaxy luminosity, although it remains detectable over the full luminosity range of our sample; and (2) the slope is stronger for GC populations located at smaller galactocentric distances. These characteristics suggest that the observed trend is, at least partially, shaped by external agents. We examine several physical mechanisms that might give rise to the observed color-magnitude relation including: (1) presence of contaminants like super-clusters, stripped galactic nuclei, or ultra-compact dwarfs; (2) accretion of GCs from low-mass galaxies; (3) stochastic effects; (4) the capture of field stars by individual GCs; and (5) GC self-enrichment. Although none of these scenarios offers a fully satisfactory explanation of the observations, we conclude that self-enrichment and field-star capture, or a combination of these processes, offer the most promising means of explaining our observations.

Subject headings: galaxies: clusters: individual: Virgo - galaxies: dwarf - galaxies: fundamental parameters - galaxies: nuclei - globular clusters: general
\end{abstract}

\section{INTRODUCTION}

It is by now an accepted paradigm that the formation of globular clusters (GCs) is closely linked to the formation of their host galaxies (e.g. Searle \& Zinn 1978, Ashman \& Zepf 1992, Kissler-Patig 1997, Hilker et al. 1999b,

\footnotetext{
${ }^{1}$ Based on observations with the NASA/ESA Hubble Space Telescope obtained at the Space Telescope Science Institute, which is operated by the association of Universities for Research in Astronomy, Inc., under NASA contract NAS 5-26555.

2 European Southern Observatory, Karl-Schwarzschild-Strasse 2, 85748 Garching bei München, Germany; smieske@eso.org, ajordan@eso.org, mkissler@eso.org

3 Astrophysics, Denys Wilkinson Building, University of Oxford, 1 Keble Road, OX1 3RH, UK

${ }^{4}$ Herzberg Institute of Astrophysics, Victoria, BC V9E 2E7, Canada; patrick.cote@nrc-cnrc.gc.ca, laura.ferrarese@nrc-cnrc.gc.ca, eric.peng@nrc-cnrc.gc.ca

5 Department of Physics and Astronomy, The Johns Hopkins University, 3400 North Charles Street, Baltimore, MD 21218-2686; jpb@pha.jhu.edu, smei@pha.jhu.edu

${ }^{6}$ Department of Physics and Astronomy, PO Box 642814, Washington State University, Pullman, WA 99164

7 Department of Physics, Rochester Institute of Technology, 84 Lomb Memorial Drive, Rochester, NY 14623; merritt@cis.rit.edu

${ }^{8}$ Institute for Astronomy, University of Hawaii, 2680 Woodlawn Drive, Honolulu, HI 96822; jt@ifa.hawaii.edu

${ }_{9}^{9}$ Gemini Observatory Southern Operations Center c/o AURA, Casilla 603 La Serena, Chile; mwest@gemini.edu
}

Forbes et al. 1997, Côté et al. 1998, Beasley et al. 2002). In recent years, observational data for extragalactic GC systems have made a huge leap forward in quality, thanks mainly to high-resolution imaging from the Hubble Space telescope, and to wide field imaging and multi-object spectroscopy from $8 \mathrm{~m}$-class ground based telescopes (see West et al. 2004 and references therein).

As a rule, studies of extragalactic GCs have tended to focus on correlations between the global photometric properties of the GC systems (GCSs) and those of their host galaxy. For instance, Peng et al. (2006), examined the color distributions for GCs belonging to 100 galaxies observed in the ACS Virgo Cluster Survey (ACSVCS; Côté et al. 2004; see also below), finding nearly all of these galaxies $\left(-22<M_{B}<-15 \mathrm{mag}\right)$ to possess bimodal, or at least asymmetric, GC color distributions. Consistent with previous findings (e.g., Gebhardt \& Kissler-Patig 1999; Kundu et al. 2001; Larsen et al. 2001), the mean GC color was found to correlate with galaxy luminosity in the same sense as the wellknown color-magnitude relation for early type galaxies (e.g. Bower, Lucey \& Ellis 1992; Karick et al. 2003; Hilker et al. 2003; Ferrarese et al. 2006). A corresponding GC color - galaxy color relation was also 
found by Peng et al. (2006), confirming previous findings (e.g., Burgarella et al. 2001; Larsen et al. 2001; Lotz et al. 2004).

At the same time, analyses of the color magnitude diagrams for the GCSs of early-type galaxies revealed some tentative evidence for a dependence of individual GC color on luminosity. Ostrov et al. (1998) found from Washington photometry of NGC 1399 that the color peaks of the GC color distribution seem to merge at the highest luminosities. Dirsch et al. (2003) confirmed this finding for the same galaxy based on an expanded sample of GCs. There have also been hints of such a behavior in other galaxies such as NGC 5128 (Harris et al. 2004) and M87 (Whitmore et al. 1995; Larsen et al. 2001).

With the improved imaging capabilities of HST provided by the Advanced Camera for Surveys (ACS; Ford et al. 1998), it has become possible to investigate this issue in more detail. Harris et al. (2006) used ACS imaging to investigate the distribution of GCs in the $(I, B-I)$ color magnitude plane for eight brightest cluster galaxies in the range $1800 \lesssim c z \lesssim 3200 \mathrm{~km} \mathrm{~s}^{-1}$. For the four galaxies with the largest number of GCs - and also the joint sample of eight GC systems - they found a significant trend towards redder colors for increasing luminosity for the subpopulation of blue GCs. This finding led them to suggest a mass-metallicity relation of the form $Z \propto M^{0.55}$ for this subpopulation. Curiously, no colormagnitude relation was found for the red GCs.

Strader et al. (2006) used the publicly available data from the ACSVCS to look for color-magnitude trends in the three brightest Virgo Cluster galaxies M49, M87 and M60. For the blue GC populations of M87 and M60, they found a significant trend of redder color with brighter GC $z$-band luminosity similar to that found by Harris et al. (2006). They too interpreted this trend as evidence for a mass-metallicity relation among the blue (metalpoor) GCs and suggested self-enrichment as a possible explanation. No significant color-magnitude trend was found for M49, the brightest member of the Virgo Cluster.

Both the Harris et al. (2006) and Strader et al. (2006) studies used heteroscedastic mixture models to fit the colors of an assumed double-Gaussian color distribution to the observed GC color-magnitude diagrams (CMDs) as a function of magnitude. Harris et al. use KMM (e.g. Ashman, Bird \& Zepf 1994), while Strader et al. use Nmix (Richardson \& Green 1997). Two important issues that were not addressed by these studies are the subject of this paper: first, we will investigate the color-magnitude correlations not only in the red (i.e., $z$ ), but also in the blue band (i.e., $g$ ); second, we will investigate how the blue peak slope varies as a function of host galaxy luminosity and galactocentric distance. Note that the Harris et al. (2006) study targeted only brightest cluster galaxies, so it spanned a relatively limited range in galaxy luminosity.

We finally point out that there is recent evidence for the existence of a GC color-magnitude relation also in spiral galaxies. Based on ACS imaging, Spitler et al. (2006) found a significant color-magnitude trend for the Sombrero galaxy (NGC 4594) implying $Z \propto M^{0.30}$, which is a somewhat weaker trend than suggested by Harris et al. (2006) and Strader et al. (2006) for giant ellipticals.
The aim of this paper is to investigate the colormagnitude trend of GCs over the entire host galaxy luminosity range covered by the ACSVCS. Special emphasis will be put on environmental dependencies of this trend and checks of the fitting technique. The paper is structured as follows: In $\$ 2$ we outline how GC candidates are selected in the ACSVCS. In $\$ 3$ we compare KMM fits to simulated CMDs with fits to observed CMDs. In $\$$ we investigate the environmental dependence of the colormagnitude trend. In $\$ 5$ we examine a variety of physical effects that may create these trends. We finish this paper with the summary and conclusions in 86

\section{SELECTION OF GLOBULAR CLUSTERS FROM THE ACSVCS}

The ACSVCS represents the most complete and homogeneous study of extragalactic GCs performed to date. The sample consists of 100 early-type (E, S0, $\mathrm{dE}, \mathrm{dE}, \mathrm{N}, \mathrm{dS} 0$ ) members of the Virgo cluster. Each galaxy was imaged in the F475W and F850LP filters $\left(\approx g_{475}\right.$ and $z_{850}$, respectively) for a total of 750 seconds and 1210 seconds, respectively. This filter combination gives roughly a factor-of-two improvement in wavelength baseline and metallicity sensitivity compared to the "canonical" $(V-I)$ color index. The identification of bona-fide GCs from these images is performed in the size-magnitude plane as described in Peng et al. (2006). This selection procedure - which is possible because the half-light radii of GCs are marginally resolved (Jordán et al. (2005)) at the distance of Virgo $(d=16.5 \mathrm{Mpc}$; Tonry et al. (2001); Mei et al. (2005)) - greatly reduces contamination from foreground stars and background galaxies.

The ACS images had been reduced using a dedicated pipeline described in detail by Jordán et al. (2004ab; Papers II and III). In brief, the reduction steps are: image combination, galaxy modeling and model subtraction, rejection of obvious background galaxies, and the measurement of magnitudes and sizes for candidate GCs using the program KINGPHOT (Jordán et al. 2005; Paper X). The result is a catalog of integrated $g$ and $z$ magnitudes, $(g-z)$ colors and half-light radii, $r_{h}$, for each candidate GC. Magnitudes and colors are corrected for foreground extinction using the reddening maps of Schlegel et al. (1998).

To estimate the contamination by background galaxies, an identical reduction procedure was applied to 17 blank high-latitude fields observed by ACS in the $g$ and $z$ filters. Because background galaxies are typically larger at comparable luminosities (or, conversely, fainter at comparable sizes), a statistical decontamination is performed in size-magnitude space (see Peng et al. 2006 and Jordán et al. 2006, in prep. for details): to this end, first a nonparametric density model is fitted to the size-magnitude distribution of sources detected in background fields. For the GC candidate magnitude distribution, a Gaussian luminosity function is assumed. The GC candidate size distribution is fitted by a non-parametric kernel estimate plus a power law tail. The resulting surface densities in the size-magnitude plane for background sources and GCs then make it possible to assign a GC probability, $\mathcal{P}_{\text {gc }}$, to every source detected in the galaxy image. All candidates with probabilities $\mathcal{P}_{\text {gc }} \geq 0.5$ are considered GCs. The remaining contamination by background 


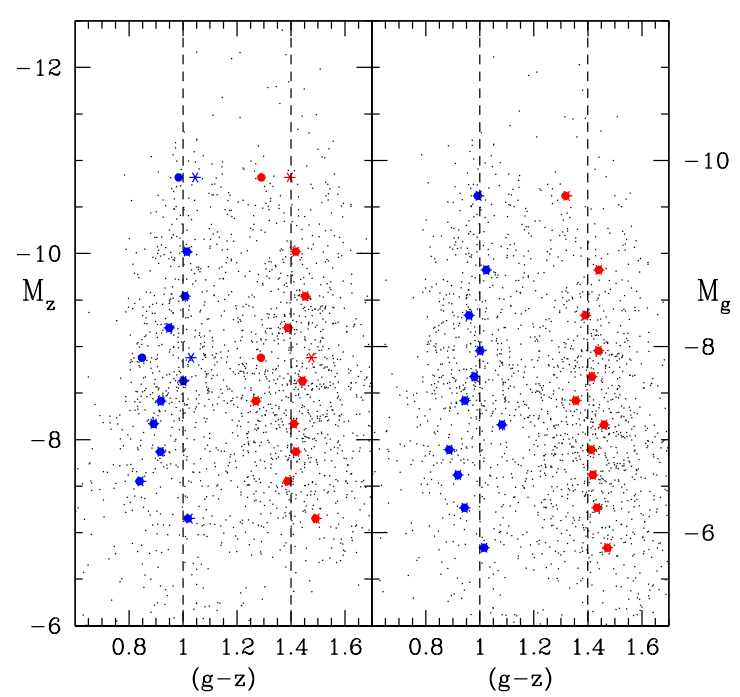

FIG. 1. - Color-magnitude diagram for GCs in M87 (= NGC4486 = VCC1316) using $M_{z}$ (left panel) and $M_{g}$ (right panel). Dashed lines at $(g-z)=1.0$ and $(g-z)=1.4$ are shown as a visual aid; they correspond to the approximate median colors of the red and blue subpopulations. Circles and asterisks indicate KMM fitting results. Circles show KMM results for initial guesses of 0.8 and 1.2 mag for the two peaks; asterisks show the results obtained for initial guesses of 1.0 and 1.4 mag.

galaxies that are assigned $\mathcal{P}_{\mathrm{gc}} \geq 0.5$ is negligible for the sake of this study. We have tested this by statistically cleaning the GC CMDs for each galaxy, using the colormagnitude distribution of sources with $\mathcal{P}_{\mathrm{gc}} \geq 0.5$ in the 17 blank background fields.

\section{ANALYSIS OF THE COLOR MAGNITUDE DIAGRAMS}

\subsection{Results from KMM}

Figure 1 shows $M_{g^{-}}(g-z)$ and $M_{z^{-}}(g-z)$ CMDs for the GCS of M87 (= NGC4486 = VCC1316), the central galaxy of the Virgo Cluster and the galaxy with the singlest largest population of GCs. For comparison, Figure 2 shows the co-added CMDs for the 79 ACSVCS galaxies that have reliable SBF distances (Mei et al. 2005, 2006) and which have a discernible GC population (see Peng et al. 2006). We use the SBF distance moduli from Mei et al. $(2005,2006)$, which have an internal precision of $\approx 0.07 \mathrm{mag}$, to assign absolute magnitudes to the associated GC candidates. To better visualize trends across host galaxy magnitude, the 79 galaxies are subdivided into four broad luminosity groups such that the number of GCs per group falls in the range $N_{\mathrm{gc}} \approx 2400$ to 3300 .

Luminosity Group 1 consists of the three brightest AVCVCS galaxies M49 (= NGC4472 = VCC1226; $M_{B}=-21.7 \mathrm{mag}$ ), M87 (= NGC4486 = VCC1316; $\left.M_{B}=-21.4 \mathrm{mag}\right)$, and M60 (= NGC4649 = VCC1978; $\left.M_{B}=-21.2 \mathrm{mag}\right)$. Group 2 consists of six ACSVCS galaxies with $-21<M_{B}<-20$ mag. Group 3 consists of 17 ACSVCS galaxies with $-20<M_{B}<-18.4$ mag. The remaining 53 ACSVCS galaxies with $-18.4<M_{B}<$ -15.2 mag make up Group 4. The three galaxies in Group 1 are all so-called "core-Sérsic galaxies" (Ferrarese et al. 2006), while those in Groups 2 and 3 are a mixture of core-Sérsic and Sérsic elliptical and lenticular galaxies. The 53 galaxies in Group 4 consist mainly, but not exclusively, of early-type dwarfs (e.g., dE, dE,N, dS0 and $\mathrm{dS} 0, \mathrm{~N})$.
We have applied KMM to the combined CMDs for these four groups, as well as to the individual CMDs for the three galaxies in Group 1. To this end, we subdivided the CMDs into luminosity bins, each bin containing the same number of data points. For the CMDs of M49 and M60, the size of each luminosity bin is 100 , for M87 the size is 150 , and for the combined CMDs the bin size is $200 \mathrm{GCs}$. KMM was then run on the GC color distribution in each luminosity bin. As in the paper by Harris et al. (2006), we use the heteroscedastic option of KMM: we allow for Gaussians of different widths to be fitted to the blue and red GC populations. The fitted mean positions are plotted over the respective CMDs in Figures [1] and 2 We show the KMM results for two different initial guesses for the mean colors of the blue and red GC populations: one for the pair $(g-z)=0.8$ and $1.2 \mathrm{mag}$, another one for $(g-z)=1.0$ and $1.4 \mathrm{mag}$. These different guesses were chosen to cover: (1) the range of peak positions between CMDs of the various luminosity groups; and (2) the range caused by the color-magnitude trend itself (see Figure 2). Linear slopes between color and absolute magnitude are obtained from ordinary least square fits to the KMM peak positions for both pairs of initial guesses, fitting color as a function of magnitude. GCs fainter than $M_{z}=-7.7 \mathrm{mag}$ and $M_{g}=-6.7 \mathrm{mag}$ were excluded from the fits, to avoid effects of photometric incompleteness on the measured slopes. The mean of both fits is adopted as the final best-fit slope. The fit errors of the slope are derived from re-sampling the points using as dispersion the observed scatter around the fitted relation. We note that this scatter is for all luminosity groups larger (by $30 \%$ for luminosity group 1 and by $100 \%$ for group 4 ) than the average statistical error estimate for the peak position, given by $\frac{\sigma}{\sqrt{N}}$. Here, $\sigma$ is the KMM width of the Gaussian peak and $N$ the average number of GCs per magnitude bin associated to the peak. We are therefore confident that our error estimates are not too optimistic. For the few cases where the difference between the results obtained using the two initial guesses was larger than the formal fit errors, we adopted the difference as the error. The resulting slopes of those fits are shown in Table 1

We first consider $M_{z}$ vs. $(g-z)$ for Group 1 . In this case, we detect a very significant slope $\gamma_{z} \equiv \frac{d(g-z)}{d z}=$ $-0.037 \pm 0.004$ for the blue peak position. When restricting the fit to $M_{z}>-10 \mathrm{mag}$ (i.e., rejecting the two brightest magnitude bins in Figure 2), the result is $\gamma_{z}=-0.033 \pm 0.007$. This is significant at the $4.5 \sigma$ level, indicating that the color-magnitude trend is not restricted to just the brightest GCs. For M87 and M60 alone, a comparable slope is derived, albeit at somewhat lower $(\approx 3 \sigma)$ significance. The slope for M49 is consistent with zero, in agreement with the findings of Strader et al. (2006). When excluding M49 from Group 1 , the slope for the blue population rises slightly to $\gamma_{z} \approx-0.040$. The non-detection of a color-magnitude trend for M49 is intriguing, since this galaxy has a similar number of GCs as M60 and is similar to M60 and M87 in terms of luminosity, color, surface brightness and GC dynamics (Peng et al. 2006; Ferrarese et al. 2006; Mei et al. 2005; Côtê et al. 2001; 2003). The fact that M49 is located at the center of its own sub-cluster - being offset by $\approx 4.5^{\circ}$ from the Virgo Cluster center - may hint at some environmental effect on the strength of the 

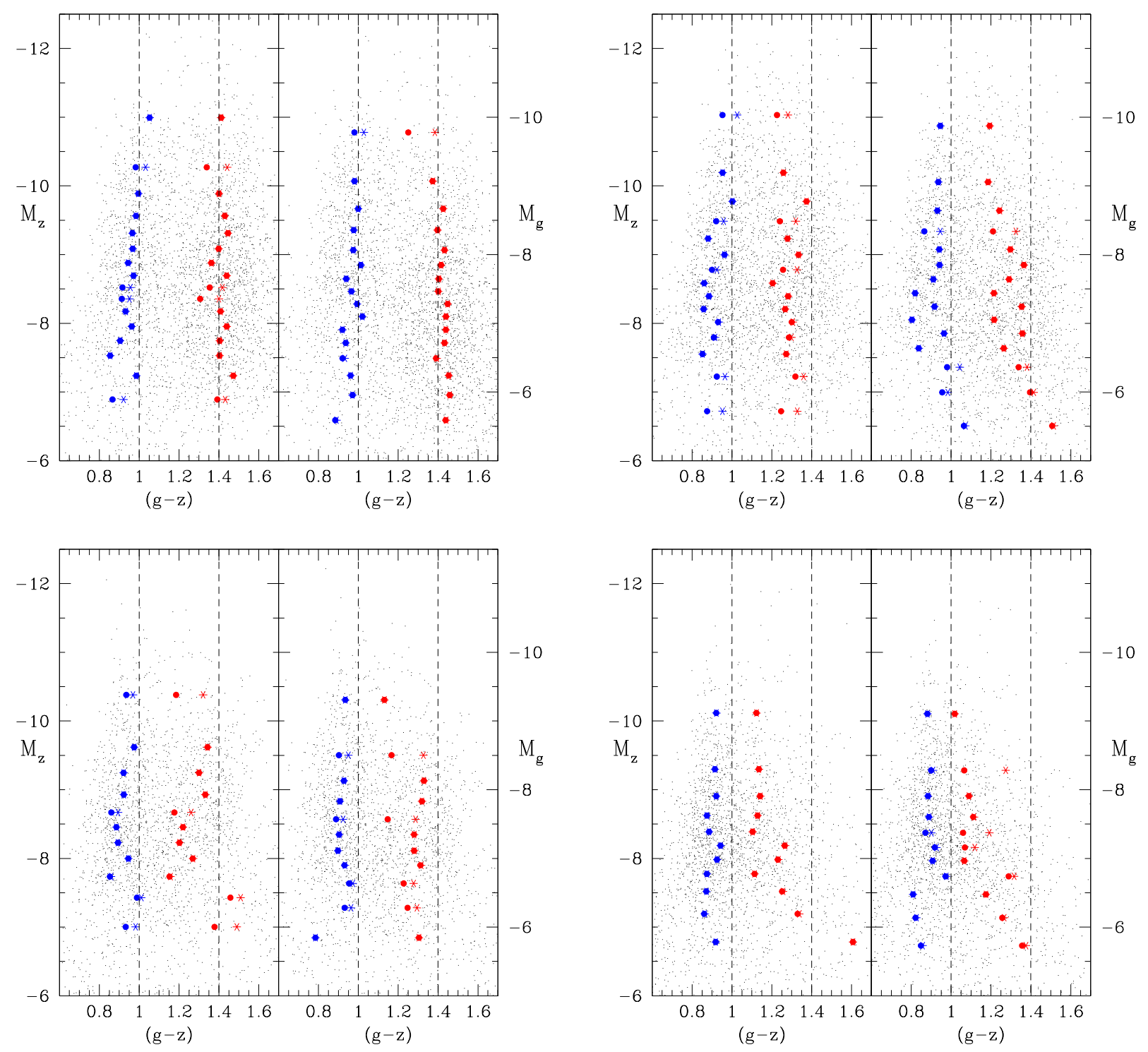

FIG. 2.- The same as Figure 1 except for the combined sample of GCs in each of the four host-galaxy luminosity groups (see Table 1 . (Upper left) The co-added CMD for GCs belonging to the three brightest ACSVCS galaxies, having $M_{B}<-21$ mag (Group 1). (Upper right) The co-added CMD for GCs belonging to the six ACSVCS galaxies with $-21<M_{B}<-20$ mag (Group 2). (Lower left) The co-added CMD for GCs belonging to the 16 ACSVCS galaxies with $-20<M_{B}<-18.4$ mag (Group 3). (Lower right) The co-added CMD for GCs belonging to 53 ACSVCS galaxies with $-18.4<M_{B}<-15.2$ mag (Group 4). The luminosity groups have been chosen such that the number of GCs in each co-added CMD are comparable (i.e., $\left.N_{\mathrm{gc}}=2400-3300\right)$.

observed color-magnitude trend.

In contrast to the strong correlation $\gamma_{z}$ exhibited by the blue GCs in Group 1, $\gamma_{z}$ is insignificant for the red GCs in this group. However, the situation changes when using $M_{g}$ instead of $M_{z}$ as magnitude in the color-magnitude diagram (see Table (1). The slope $\gamma_{g} \equiv \frac{d(g-z)}{d g}$ for the blue population is more than $50 \%$ smaller than $\gamma_{z}$ and, as a result, has a lower level of significance $(1.7 \sigma$.) Considered individually, $\gamma_{g}$ of the blue population becomes insignificant for all three galaxies in this group. In turn, the slope $\gamma_{g}$ of the red population becomes marginally positive - i.e., opposite in sense to that defined by $\gamma_{z}$ for the blue population.

\subsection{Monte Carlo Simulations to test KMM}

The difference in slopes between $z$ and $g$ raises the question as to what the "true" slope actually is. To in- vestigate this issue in more detail, we compare in Figure 3 the actual measurements for Group 1 with two sets of 20 Monte-Carlo simulations for the M87 CMD. The first set of CMDs has an implemented slope $\gamma_{z}=-0.040$ for the blue $\mathrm{GCs}^{10}$ and $\gamma_{z}=0$ for the red ones; the other set has $\gamma_{z}=0$ implemented for either GC population. These artificial CMDs were created by assuming the average peak widths derived by KMM for the blue and red GCs, taking into account also the photometric errors on the colors. The luminosity function is sampled from a kernel estimator of the original CMD of M87, separately for each color peak. Figure 4 compares the real and simulated M87 CMD with an implemented slope. Note an important feature of those CMDs: the red peak is populated to

10 Note that the slope $\gamma_{g}$ corresponding to $\gamma_{z}=-0.040$ is of course not identical to $\gamma_{z}$, but this difference is negligible for the sake of this study: $\gamma_{z}=-0.040 \neq \gamma_{g}=\gamma_{z}+\gamma_{z}^{2}=-0.0384$ 

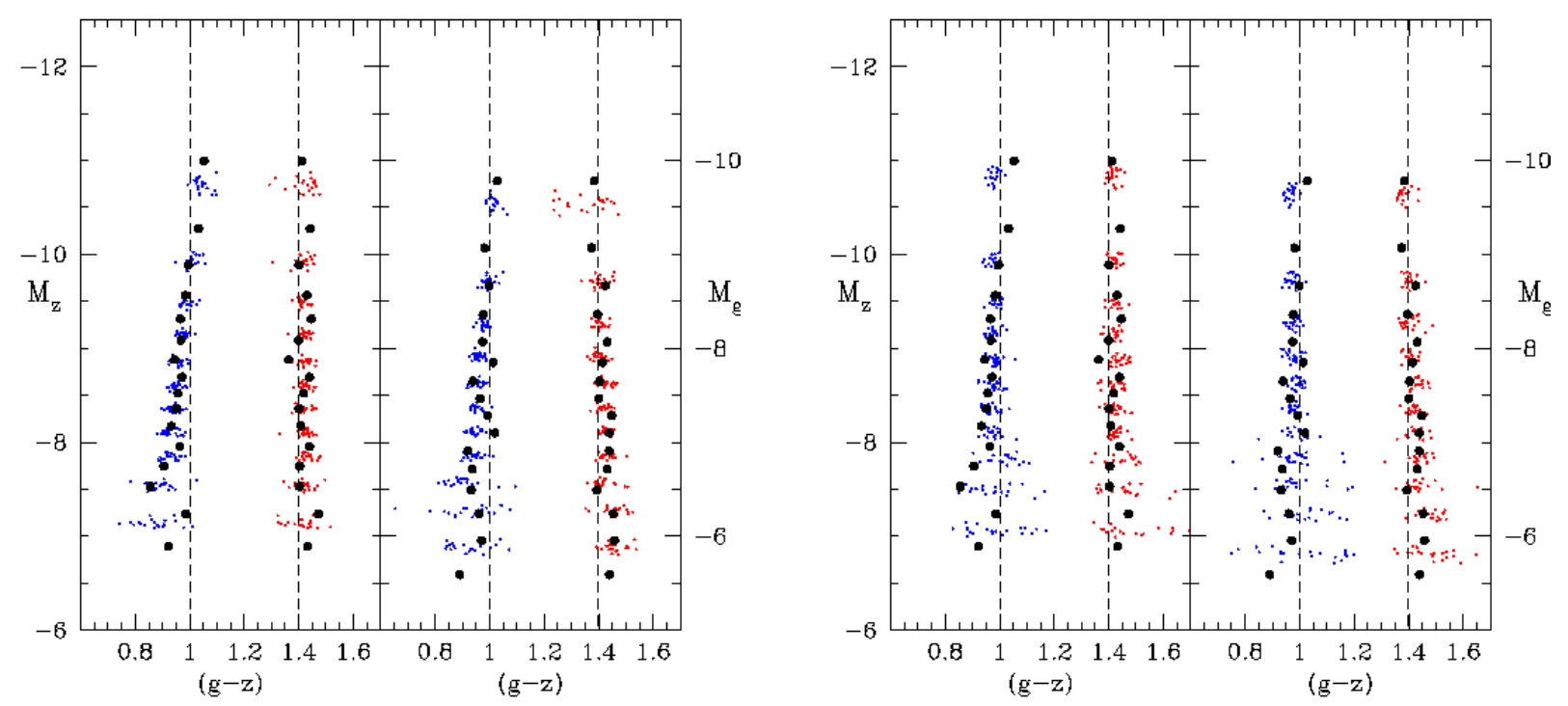

FIG. 3. - KMM result for the co-added CMD of the three brightest ACSVCS galaxies (Group 1, see Figure 2 and Table 1) compared to KMM results for simulated M87 CMDs. Filled circles indicate KMM fitting results for the real data with initial guesses of 1.0 and 1.4 mag for peak positions (see Figures 1 and 2]. Dots indicate KMM peak fittings for 20 simulated CMDs. (Left Panel) Simulated CMDs with a slope of $\gamma_{z} \equiv \frac{d(g-z)}{d z}=-0.04$ implemented for the blue subpopulation; no correlation has been implemented for the red subpopulation. Note the artificial bending of the red sequence toward blue colors at bright luminosities, especially for $M_{g}$. (Right Panel) Simulated CMD with no color-magnitude relation implemented for either population.
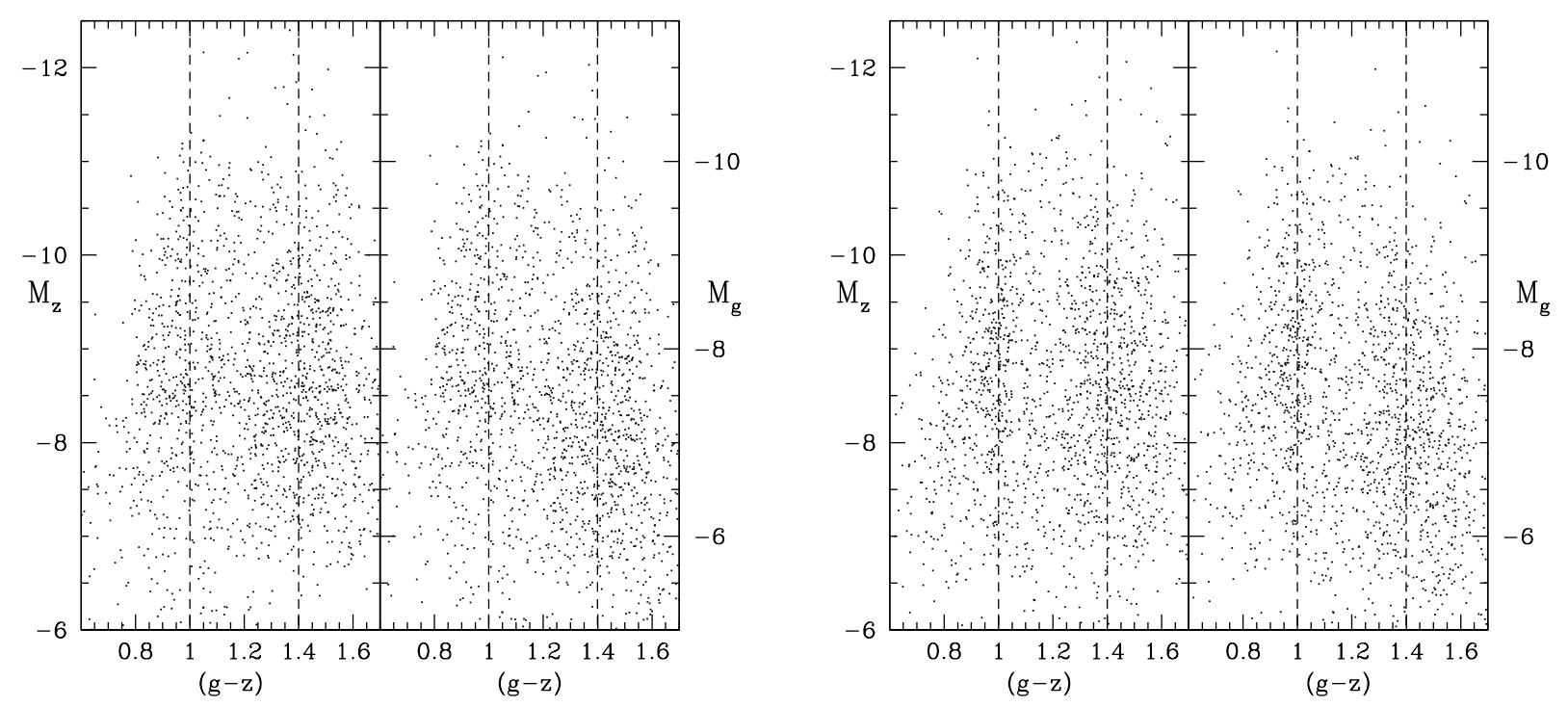

FIG. 4.- Comparison between real (left panel) and simulated (right panel) CMD for GCs in M87. The simulated CMD has a slope of $\gamma_{z}=-0.04$ input for the blue subpopulation.

brighter absolute luminosities in $M_{z}$ than in $M_{g}$, while the blue peak does not show such a difference. This is because, relative to GCs in the blue population, GCs in the red population are brighter in $M_{z}$ than in $M_{g}$.

Table 1 gives the slopes fitted to the mean colors from KMM for the two simulated sets of M87 CMDs, together with the slopes for the real data. This comparison shows that both the CMD of M87 alone, and that for the coadded CMD of Group 1, are inconsistent with a magnitude independent color for the blue GCs. The data are consistent with a simulated slope of -0.040 for the blue GCs and no slope for the red GCs.

Note the positive slope found for the red GCs in both the real and simulated data in $M_{g}$. Averaging over the
20 simulated CMDs, the mean red slope of $\gamma_{g} \simeq+0.03$ is different from zero at the $5.7 \sigma$ level. That finding is noteworthy since the simulations do not implement a slope for the red peak. This exercise therefore demonstrates an important caveat of the KMM fitting: at a given magnitude, as one of the two peaks becomes de-populated (in this case the red peak in $M_{g}$ ), its KMM position will artificially be dragged towards the position of the more prominent peak. As a consequence, the mean color of the blue population will be artificially shifted towards bluer colors. This results in a weaker slope for the blue peak in $M_{g}$ than in $M_{z}$. In $M_{z}$, both peaks are populated up to comparable absolute luminosities, and therefore the fitted slopes for both red and blue peaks correspond to 
TABLE 1

Color-magnitude trends for Red and blue globular Clusters in ACS Virgo Cluster Survey galaxies, Determined with KMM FITS

\begin{tabular}{|l|rrrr|}
\hline \hline Sample & $\gamma_{z, \text { blue }}$ & $\gamma_{z, \text { red }}$ & $\gamma_{g, \text { blue }}$ & $\gamma_{g, \text { red }}$ \\
\hline M49 $\left(M_{B}=-21.7\right)$ & $-0.008 \pm 0.024$ & $0.013 \pm 0.018$ & $0.021 \pm 0.018$ & $0.043 \pm 0.008$ \\
M87 $\left(M_{B}=-21.4\right)$ & $-0.042 \pm 0.015$ & $0.003 \pm 0.023$ & $-0.016 \pm 0.023$ & $0.025 \pm 0.017$ \\
M60 $\left(M_{B}=-21.2\right)$ & $-0.028 \pm 0.009$ & $0.012 \pm 0.018$ & $-0.003 \pm 0.033$ & $0.072 \pm 0.017$ \\
\hline Simulated M87 NoCM & $0.002 \pm 0.012$ & $0.010 \pm 0.015$ & $0.008 \pm 0.015$ & $0.028 \pm 0.022$ \\
Simulated M87 CM & $-0.039 \pm 0.009$ & $0.010 \pm 0.014$ & $-0.028 \pm 0.010$ & $0.031 \pm 0.024$ \\
\hline Group1 $\left(-21.7<M_{B}<-21\right)$ & $-0.037 \pm 0.004$ & $-0.001 \pm 0.007$ & $-0.015 \pm 0.009$ & $0.032 \pm 0.019$ \\
Group2 $\left(-21<M_{B}<-20\right)$ & $-0.033 \pm 0.011$ & $0.001 \pm 0.014$ & $-0.022 \pm 0.016$ & $0.042 \pm 0.018$ \\
Group3 $\left(-20<M_{B}<-18.4\right)$ & $-0.032 \pm 0.012$ & $-0.043 \pm 0.024$ & $-0.010 \pm 0.007$ & $0.055 \pm 0.029$ \\
Group4 $\left(-18.4<M_{B}<-15.2\right)$ & $-0.009 \pm 0.013$ & $0.028 \pm 0.028$ & $0.025 \pm 0.012$ & $0.058 \pm 0.040$ \\
Group1, inner regions & $-0.061 \pm 0.014$ & $-0.006 \pm 0.014$ & $-0.007 \pm 0.018$ & $0.059 \pm 0.018$ \\
Group1, outer regions & $-0.026 \pm 0.008$ & $0.003 \pm 0.020$ & $-0.004 \pm 0.015$ & $0.050 \pm 0.014$ \\
Group1+2, inner regions & $-0.066 \pm 0.007$ & $-0.024 \pm 0.006$ & $-0.022 \pm 0.020$ & $0.043 \pm 0.018$ \\
Group1+2, outer regions & $-0.023 \pm 0.013$ & $0.009 \pm 0.013$ & $-0.018 \pm 0.007$ & $0.029 \pm 0.013$ \\
\hline \hline
\end{tabular}

Notes: For the samples in column 1, columns 2 to 5 give the slopes $\gamma$ between $(g-z)$ and the respective magnitude of the blue and red GC subpopulations, as derived from KMM determined peak positions. Errors come from random resampling of the data points using their measured dispersion around the fit. For the two simulated sets of CMDs, "CM" refers to an implemented slope $\gamma_{z}=-0.040$ in the blue peak and no slope in the red peak. "NoCM" refers to no implemented slope. Errors quoted for the simulations are rms around the mean for 20 simulated M87 CMDs. The faint limiting magnitude for fitting was -7.7 mag in $M_{z}$ and $-6.7 \mathrm{mag}$ in $M_{g}$. The separation between inner and outer region for the lower four samples was defined at a galactocentric distance of $65^{\prime \prime}$. By that, the inner and outer sample were of approximately equal size.

TABLE 2

Color-magnitude trends for Red and blue globular Clusters in ACS Virgo Cluster Survey galaxies, Determined with BIWEIGHT FITS

\begin{tabular}{|l|rrrr|}
\hline \hline Sample & $\gamma_{z, \text { blue }}$ & $\gamma_{z, \text { red }}$ & $\gamma_{g, \text { blue }}$ & $\gamma_{g, \text { red }}$ \\
\hline M49 $\left(M_{B}=-21.7\right)$ & $-0.004 \pm 0.002$ & $0.003 \pm 0.003$ & $-0.005 \pm 0.006$ & $0.010 \pm 0.004$ \\
M87 $\left(M_{B}=-21.4\right)$ & $-0.031 \pm 0.007$ & $0.013 \pm 0.005$ & $-0.031 \pm 0.013$ & $0.017 \pm 0.006$ \\
M60 $\left(M_{B}=-21.2\right)$ & $-0.024 \pm 0.006$ & $0.001 \pm 0.005$ & $-0.027 \pm 0.007$ & $0.017 \pm 0.002$ \\
\hline Simulated M87 NoCM & $0.008 \pm 0.007$ & $-0.001 \pm 0.005$ & $0.008 \pm 0.004$ & $0.009 \pm 0.007$ \\
Simulated M87 CM & $-0.029 \pm 0.006$ & $0.013 \pm 0.004$ & $-0.026 \pm 0.007$ & $0.022 \pm 0.003$ \\
\hline Group1 $\left(-21.7<M_{B}<-21\right)$ & $-0.025 \pm 0.002$ & $0.009 \pm 0.003$ & $-0.027 \pm 0.004$ & $0.021 \pm 0.004$ \\
Group2 $\left(-21<M_{B}<-20\right)$ & $-0.025 \pm 0.004$ & $0.017 \pm 0.005$ & $-0.023 \pm 0.003$ & $0.025 \pm 0.006$ \\
Group3 $\left(-20<M_{B}<-18.4\right)$ & $-0.020 \pm 0.002$ & $-0.003 \pm 0.009$ & $-0.001 \pm 0.005$ & $0.018 \pm 0.010$ \\
Group4 $\left(-18.4<M_{B}<-15.2\right)$ & $-0.019 \pm 0.004$ & $0.011 \pm 0.007$ & $-0.021 \pm 0.004$ & $0.012 \pm 0.012$ \\
Group1, inner regions & $-0.035 \pm 0.005$ & $0.012 \pm 0.004$ & $-0.043 \pm 0.006$ & $0.022 \pm 0.010$ \\
Group1, outer regions & $-0.022 \pm 0.004$ & $0.003 \pm 0.009$ & $-0.020 \pm 0.005$ & $0.016 \pm 0.004$ \\
Group1+2, inner regions & $-0.032 \pm 0.004$ & $0.016 \pm 0.002$ & $-0.029 \pm 0.006$ & $0.026 \pm 0.004$ \\
Group1+2, outer regions & $-0.025 \pm 0.005$ & $0.010 \pm 0.006$ & $-0.022 \pm 0.003$ & $0.017 \pm 0.007$ \\
\hline \hline
\end{tabular}

Notes: Analogous to Table 1 Difference is that peak positions are now defined by the biweight of the color distribution blueand redwards of a luminosity independent limiting color, see Fig. 5] Fig. 6 graphically compares the fits to KMM and biweight peak positions for all four luminosity groups.

the simulated ones. That is to say, only the weaker slope in $M_{g}$ is caused by a KMM bias. KMM does not artificially introduce the very significant negative slope in the blue $M_{z}$ peak: this is clearly a real trend that is intrinsic to the data.

When interpreting the slope as a mass-metallicity relation for GCs in the blue peak, we derive a scaling $Z \propto M^{0.48 \pm 0.08}$ by combining the slope $\gamma_{z}=-0.037$ found in the previous section with

$$
\frac{d[\mathrm{Fe} / \mathrm{H}]}{d(g-z)}=5.14 \mathrm{dex} \mathrm{mag}^{-1}
$$

from Peng et al. (2006) and assuming a constant massto-light ratio over the luminosity range investigated. This is in good agreement with the scaling relation $Z \propto M^{0.55}$ found by Harris et al. (2006).

As an alternative to KMM, we have calculated the biweight location (Beers et al. 1990) for the GC subpopula- 
tions blue-wards and red-wards of a magnitude independent limiting color. This limiting color is the mean of the KMM position of the blue and red populations, weighted by the average width of either peak. The results for this alternative method are shown in Table2 As an example, we show in Fig. [5 the peak positions for Groups 1 and 4 in $M_{z}$. Generally speaking, the errors for this biweight method decrease significantly compared to the KMM data points, and the results confirm the significant slope found for the blue GC population. They also confirm that no significant correlation is found for M49. Table2 and Figure 6] show that the difference between fits in $M_{g}$ and $M_{z}$ is considerably smaller than in the case of KMM.

Nevertheless, this alternative method is itself not immune to biases, as is clear when it is applied it to the simulated CMDs (see Table 2 and Figure 6). In the case of the blue population exhibiting a negative slope, a fixed limiting magnitude between both populations will systematically decrease the absolute slope of the blue population, and introduce an artificial positive slope for the red.

\section{ENVIRONMENTAL EFFECTS}

Having examined how specific features of the CMD may influence the fitted color-magnitude relations for the blue and red GC populations, we now investigate the extent to which possible environmental effects may influence the observed trends.

\subsection{Dependence on Host Galaxy Luminosity}

Figure 2 shows CMDs for Groups 1-4 with the KMM peaks over-plotted. Derived slopes for the colormagnitude relations in each group are presented in Tables [1 and 2] The dependence of slope on host galaxy magnitude is illustrated in Figure 6

The KMM fits in $M_{z}$ show that the slope of the blue GCs in Groups 2 and 3 are indistinguishable from that for Group 1; in all three groups, the slope is inconsistent with zero at about the $3 \sigma$ level. This indicates that the color-magnitude trend among metal-poor GCs is not restricted to just the brightest galaxies. Only for Group 4 does the measured slope of the blue population become consistent with zero. The biweight fits in the lower panels of Figure 6 paint a similar picture: the slope decreases only gradually from Group 1 to 4 , and apparently does not drop to zero. Given that the biweight fits systematically underestimate the blue slope, this is an indication that the color magnitude trend persists over the entire magnitude range of the ACSVCS galaxies.

The trend for the red GCs determined using KMM becomes progressively more uncertain for fainter galaxy magnitudes. This is because of the decreasing fraction of red (metal-rich) GCs with decreasing host galaxy luminosity (Peng et al. 2006). The firmest result from Figure 6 regarding the color-magnitude relation for red GCs is that there is no strong $M_{z}$ slope for the two brightest groups; the slopes for the two faintest galaxy groups have large uncertainties. The large positive slopes found in $M_{g}$ are particularly prone to KMM biases, a conclusion that is confirmed by looking at the more robust biweight fit results. The comparison between the biweight fits to the real data and the simulations shows that the slope of the red population is consistent with zero over the full luminosity range.

\subsection{Dependence on Galactocentric Distance}

An interesting feature of the observed color-magnitude relations is illustrated in Figure 7 Here we subdivide the GC samples of Groups 1 and 2 by galactocentric radius into equally-sized, "inner" and "outer" subsamples. As the dividing radius, we choose $R=65^{\prime \prime}$, which translates to a physical distance of $\approx 5.2 \mathrm{kpc}$ at the distance of the Virgo Cluster. In terms of the effective radii, $R_{e}$, of the host galaxies in Groups 1 and 2, this fixed dividing radius corresponds to $\approx 0.3-0.6 R_{e}$ (Ferrarese et al. 2006). Tables 11 and 2 report the slopes for the different subsamples. For Group 1, the slope found for the inner GCs is a factor of $\approx 2-3$ higher than that for the outer GCs. Both the KMM and biweight methods support this conclusion, at significance levels of $2.2 \sigma$ and $2.5 \sigma$, respectively. For the combined Group 1+2 samples, the KMM fits still obtain a difference of about a factor of three (at $2.9 \sigma$ significance), while the differences are almost insignificant for the biweight fits.

In Figure 8, we plot color vs. half-light radius for GCs with $-11.5<M_{z}<-10 \mathrm{mag}$, separated into inner and outer samples. There is a small size offset between the inner and outer sample, in the sense that outer GCs are larger by about $10 \%$. This dependence of $r_{h}$ on galactocentric radius has already been investigated by Jordán et al. (2005). In Figure 9] we plot the color histograms for two luminosity ranges: in the left panel, we show GCs in the same luminosity range as in Figure 8 in the right panel, we show GCs in the range $-10<M_{z}<-9$ mag. Clearly, the gap between the two peaks is larger among the fainter sample of GCs, a consequence of the colormagnitude trend. Furthermore, for the brighter GCs, the inner and outer color histograms appear to differ more than for the fainter GCs. The KS probability with which the inner and outer sample of the bright GCs have the same underlying distribution is $1 \%$, while it is $14 \%$ for the faint sample. Indeed, while the bimodality feature is still notable for the outer bright GCs, it is slightly smeared out for the bright, inner GCs. This suggests that the dependence of the color-magnitude relation on galactocentric distance is driven mainly by the bright GCs $\left(M_{z}<-10 \mathrm{mag}\right)$.

Figure 9 also shows that the fraction of blue-to-red GCs decreases slightly for smaller galactocentric distance (see also, e.g., Dirsch et al. 2003). The corresponding fractions of blue-to-red GCs are 0.45:0.55 and 0.50:0.50 for the inner and outer GCs, respectively. It is apparent from the color histograms in Figure 9] that this small effect cannot be responsible for the steeper color-magnitude relation found for the inner sample of GCs.

\subsection{Comparison to CMDs of Other Stellar Systems}

Before proceeding, we pause to compare the distribution of GCs in the CMD with those of other stellar systems: i.e., the nuclei of ACSVCS galaxies, the ACSVCS galaxies themselves, and some candidate ultra-compact dwarf galaxies (UCDs) identified in the course of the ACSVCS.

Figure 10] compares the combined CMD of GCs (red circles), compact stellar nuclei (asterisks; Côté et al. 2006), ACSVCS galaxies (green circles; Ferrarese et al. 2006) and UCDs (open squares; Haşegan et al. 2005). In this figure, we have converted from $M_{z}$ 

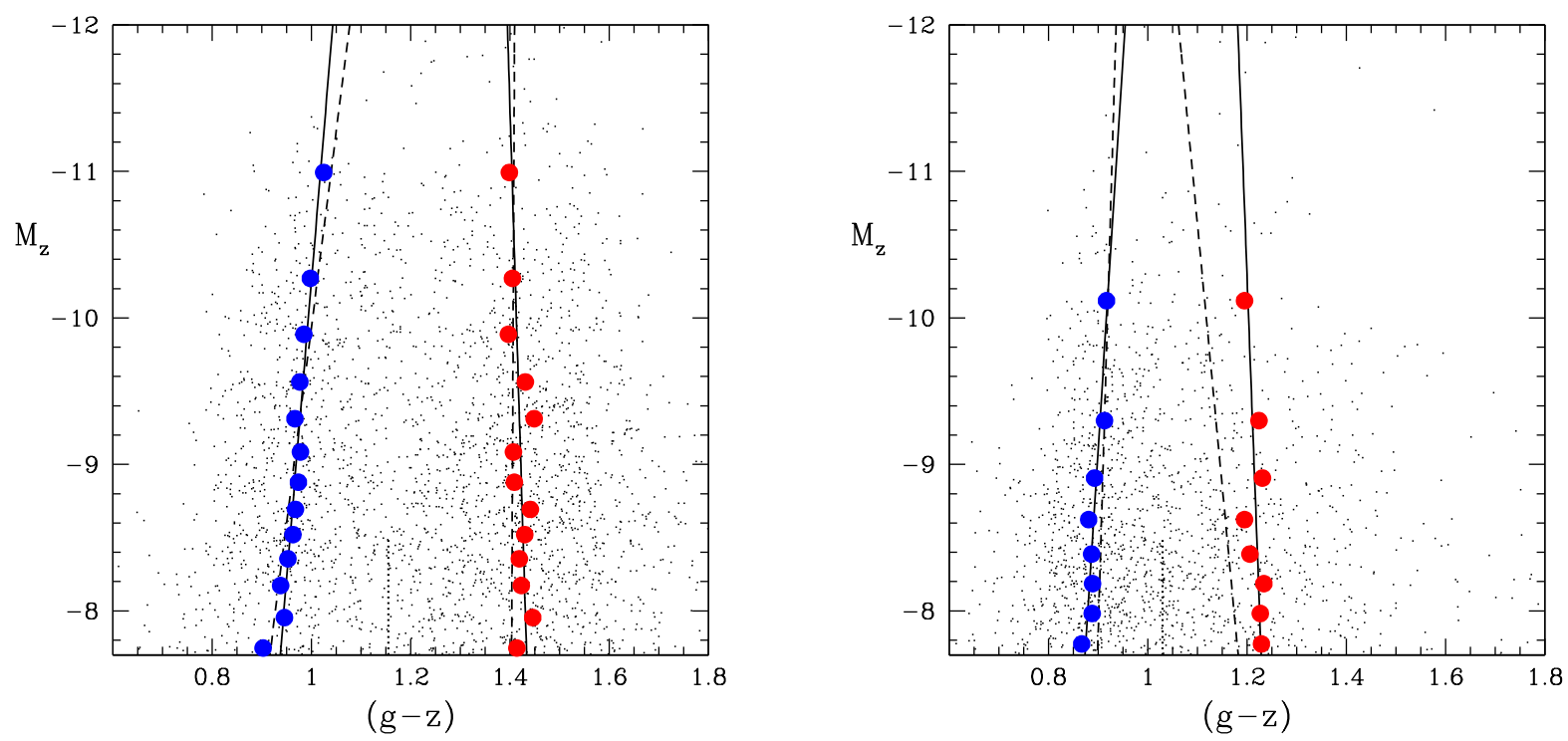

FIG. 5.- (Left Panel) Color-magnitude diagram in $M_{z}$ for GCs in Group 1. Filled circles indicate the biweight estimates for the mean GC color bluewards and redwards of the dividing color $(g-z)=1.155$, which is indicated as a dotted vertical tick mark. The solid lines are least-squares fits to the circles. For comparison, the dashed lines give the linear fit obtained from the KMM colors (Table 1). (Right Panel) Same as in the left panel, but for Group 4. The offset between the KMM and biweight fits for the red subpopulation is due to its asymmetric color distribution (i.e., an extended tail towards red colors).
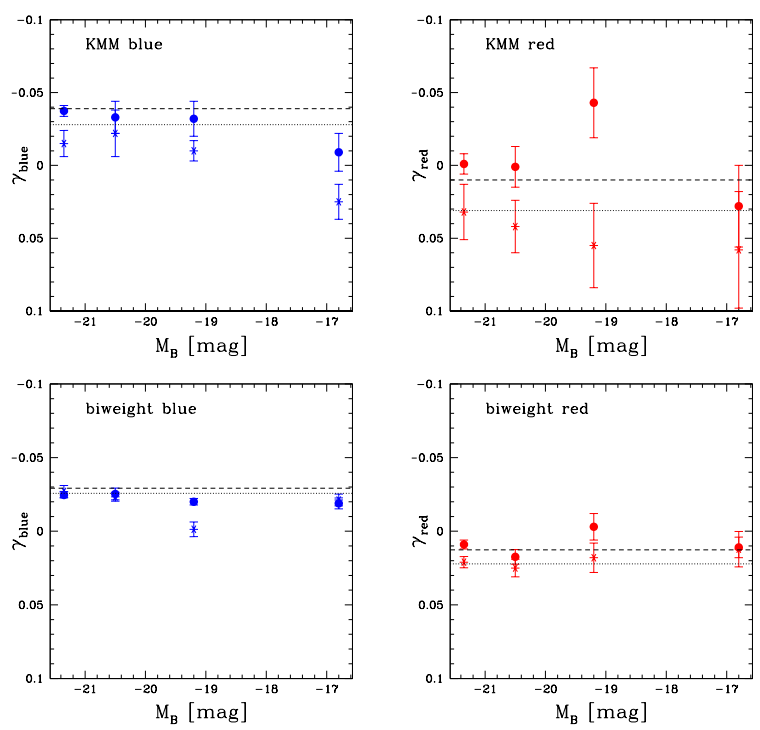

FIG. 6. - Slopes $\gamma$ of the GC color-magnitude relation for the four host-galaxy luminosity groups plotted against the respective mean host galaxy magnitude. Filled circles and asterisks show results for $\gamma_{z}$ and $\gamma_{g}$, respectively. (Upper Panels) KMM fits (see Table 1). (Lower Panels): Biweight fits (see Table 2). (Left Panels) Slopes for the blue subpopulations. (Right Panels) Slopes for the red subpopulations. The horizontal lines indicate the respective fit results to the artificial CMD of M87, with a slope of $\gamma_{z}=-0.04$ implemented for the blue subpopulations and zero slope for the red subpopulations. Dashed and dotted lines correspond to $\gamma_{z}$ and $\gamma_{g}$, respectively.

to stellar masses using the population synthesis models of Bruzual \& Charlot (2003) under the assumption of a 10Gyr old population and a Chabrier IMF (Chabrier 2003). For comparison, we also show the color-magnitude relation for early-type galaxies in the Fornax Cluster from Hilker et al. (2003), after converting from $(V-I)$ to $(g-z)$ using the transformation of Fukugita et al. (1996).

The majority of the blue GCs are not consistent with an extrapolation of the galaxy color-magnitude rela- tion, nor with that of the nuclei. Moreover, the colormagnitude relations of the nuclei and the galaxies are steeper than that of the GCs. Only the faintest nuclei appear to be roughly consistent with the color-magnitude relation observed for the blue GCs. In any case, we point out that the existence of a color-magnitude trend for the blue GCs results in a paucity of GCs at high luminosities and blue colors: i.e., $M_{z} \lesssim-10 \mathrm{mag}$ and $(g-z) \lesssim 0.9$ mag. It is difficult to understand how contamination of the observed CMDs by some unrecognized stellar system - stripped nuclei, ultra-compact dwarfs (UCDs), or otherwise - can lead to an underpopulation of this region of the CMD.

\section{DISCUSSION}

As a starting point for this discussion, we summarize the two main findings thus far, both of which concern dependencies of the measured color-magnitude slope on external factors: (1) The slope for the blue GCs decreases gradually towards fainter host galaxy luminosities, whereas there is no significant color-magnitude relation for the red GCs; and (2) the slope is steeper for GCs which lie closer to the centers of their host galaxies.

We now discuss a number of scenarios that that might help explain the observed trend: (1) contamination by super star clusters, stripped nuclei, or UCDs; (2) accretion of GCs from low-mass galaxies; (3) stochastic effects; (4) capture of field stars by individual GCs; and (5) GC self enrichment. In $\$ 5.6$ we briefly discuss how the observed color-magnitude trends appear after transforming into the metallicity-mass plane.

\subsection{Super Star Clusters, Stripped Nuclei, and UCDs}

One idea that may explain the slope difference between inner and outer GCs is the presence of overluminous star clusters of intermediate color that may lie close to the galaxy centers. Such a putative population would populate the region between the two color 

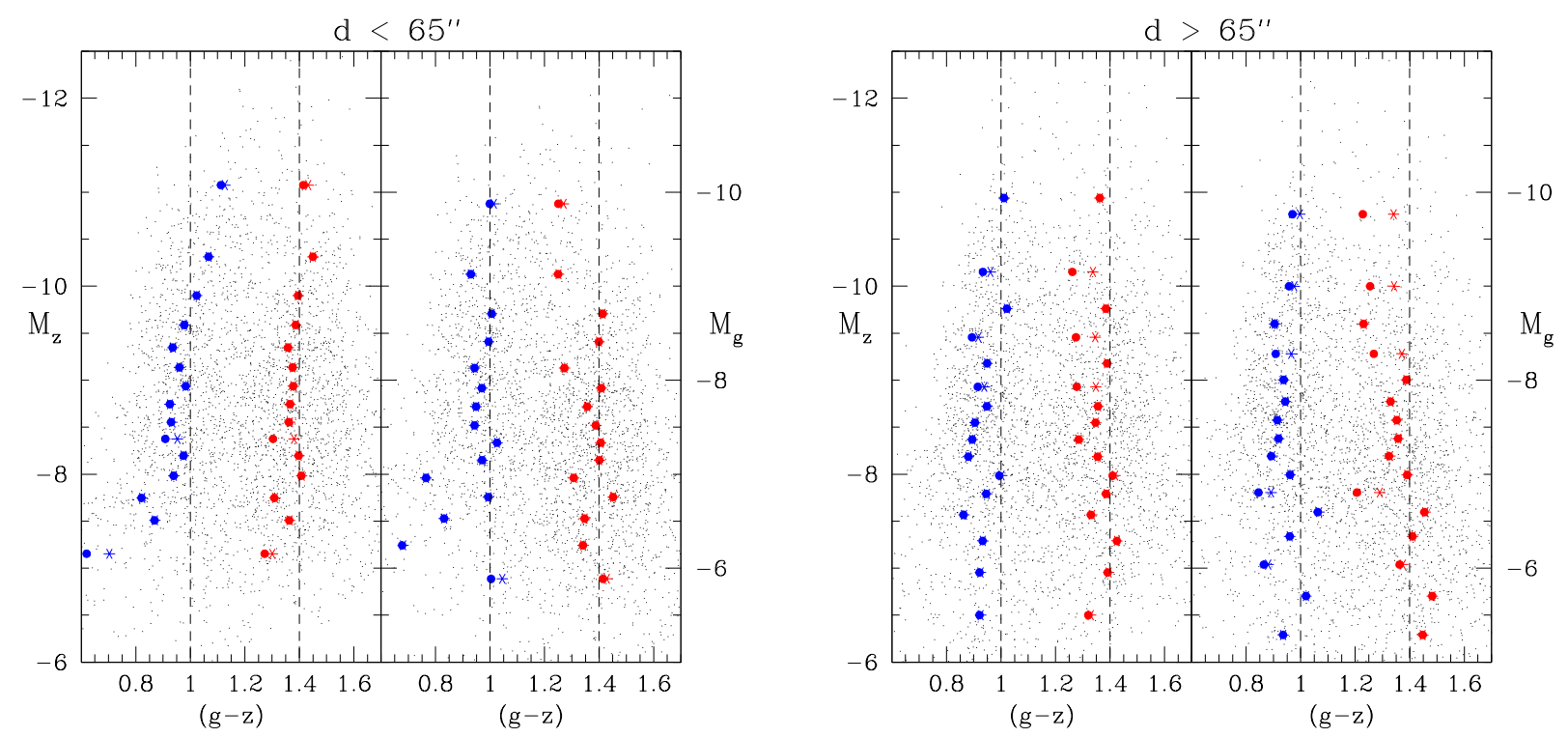

FIG. 7.- Radial dependence of the color-magnitude for the combined GC systems of Groups 1 and 2. (Left Panel) GCs having galactocentric distances $R<65^{\prime \prime}$ (corresponding to $d \lesssim 5.2 \mathrm{kpc}$ ). (Right Panel) GCs having galactocentric distance $R>65^{\prime \prime}$. Note the stronger trend for the blue subpopulation inside $65^{\prime \prime}$ (see also Table 1 .

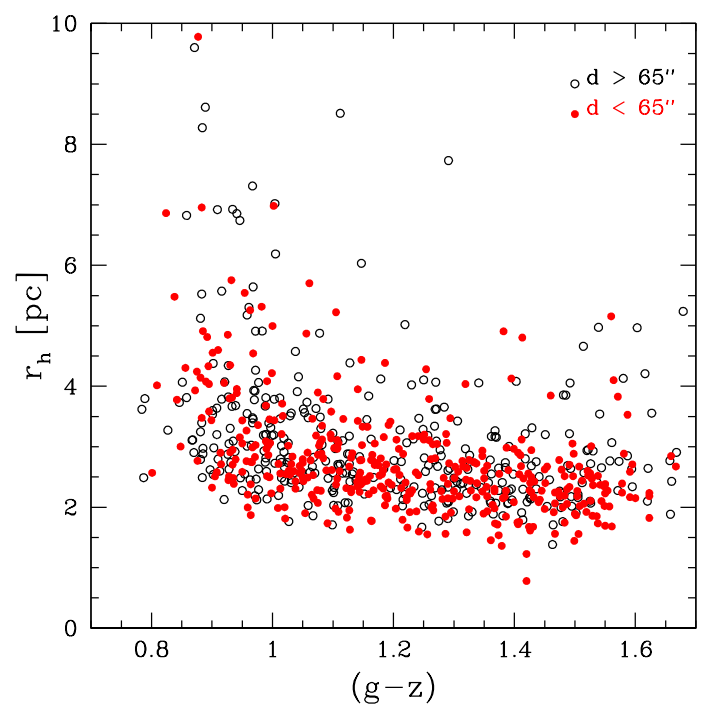

FIG. 8.- Half-light radius, $r_{h}$, plotted against $(g-z)$ for GCs belonging to Group 1 and 2 , and with magnitudes in the range $-11.5<M_{z}<-10 \mathrm{mag}$ (see also Figure $\mathbf{7 1}$. Open circles are GCs with galactocentric distances $R>65^{\prime \prime}$, filled circles are GCs with $R<65^{\prime \prime}$. The mean size of the outer GCs is larger than that of the inner GCs by about $10 \%$ (see also Jordán et al. 2005). The cumulative $r_{h}$ distributions of inner and outer GCs are inconsistent with each other at the $99.99 \%$ confidence level according to a KS test.

sequences at high luminosities. Merged super star clusters (e.g., Fellhauer \& Kroupa 2002, 2005; Kissler-Patig et al. 2006) could, in principle, be those objects. They are expected to be more extended than their monolithic counterparts of equal mass (e.g., Bekki et al. 2004; Fellhauer \& Kroupa 2002; Bastian et al. 2005). However, in the size vs. color plot shown in Figure 8 it is the outer GCs that are slightly larger. More importantly, there is no offset in the size distribution for the inner GCs with intermediate colors (i.e., $\approx(g-z)=1.20 \mathrm{mag}$ ). This makes the presence of a large number of merged star clusters unlikely.

Another possibility is the presence of "naked" or tidally stripped dE,N nuclei, as suggested by Harris et al. (2006) (see also Bassino et al. 1994). The dE,N nuclei of Virgo Cluster galaxies do exhibit a color-magnitude trend and have colors comparable to those of the blue GCs (see, e.g., Lotz et al. 2004; Côté et al. 2006; Figure 10). Côté et al. (2006) find a median half-light radius of $r_{h}=3.2 \pm 0.6 \mathrm{pc}$ for nuclei with $-11.5<M_{z}<-10 \mathrm{mag}$. This is comparable to the sizes of GCs in this same luminosity range (Figure 8). Stripped nuclei might therefore be confused with "normal" blue GCs at the highest luminosities.

However, as pointed out in 4.3 the overall distribution of GCs with the CMD shows a lack of bright, blue objects. Very generally, this dearth is not explained by the addition of an "extra" population of contaminators, but rather requires an underlying trend for most of the objects. Stripped nuclei would have to dominate the population of blue GCs. This seems unlikely to us for several reasons. First, the nuclei luminosity function peaks $\approx 2$ 4 mag brighter than that of GCs (see Lotz et al. 2004; Côté et al. 2006, see also Figure 100. More seriously, though, is the shear number of nuclei that would be required in this scenario. Observationally, there is an upper limit on the number of naked nuclei brighter than $M_{V} \approx-11$ mag from Jones et al. (2006). These authors found nine compact objects - UCDs in their notation - with $M_{B}<-10.7 \mathrm{mag}$ in a survey that is almost complete within the central degree of the Virgo Cluster (excluding the 1-2' surrounding M87). Their nine compact objects are distributed over an area that is $\sim 100 \times$ the ACS field of view, in qualitative agreement with the predictions by Bekki et al. (2003a) for the spatial distribution of tidally stripped dE,N nuclei. Therefore, a presence of dozens, or possibly hundreds, of naked nuclei in our ACS fields for the most luminous galaxies seems unlikely.

A final note regarding the possibility of UCDs as contaminators: the two most discussed formation channels for these compact stellar systems are stripped dE, N nuclei and merged stellar super-clusters (e.g. Minniti et 


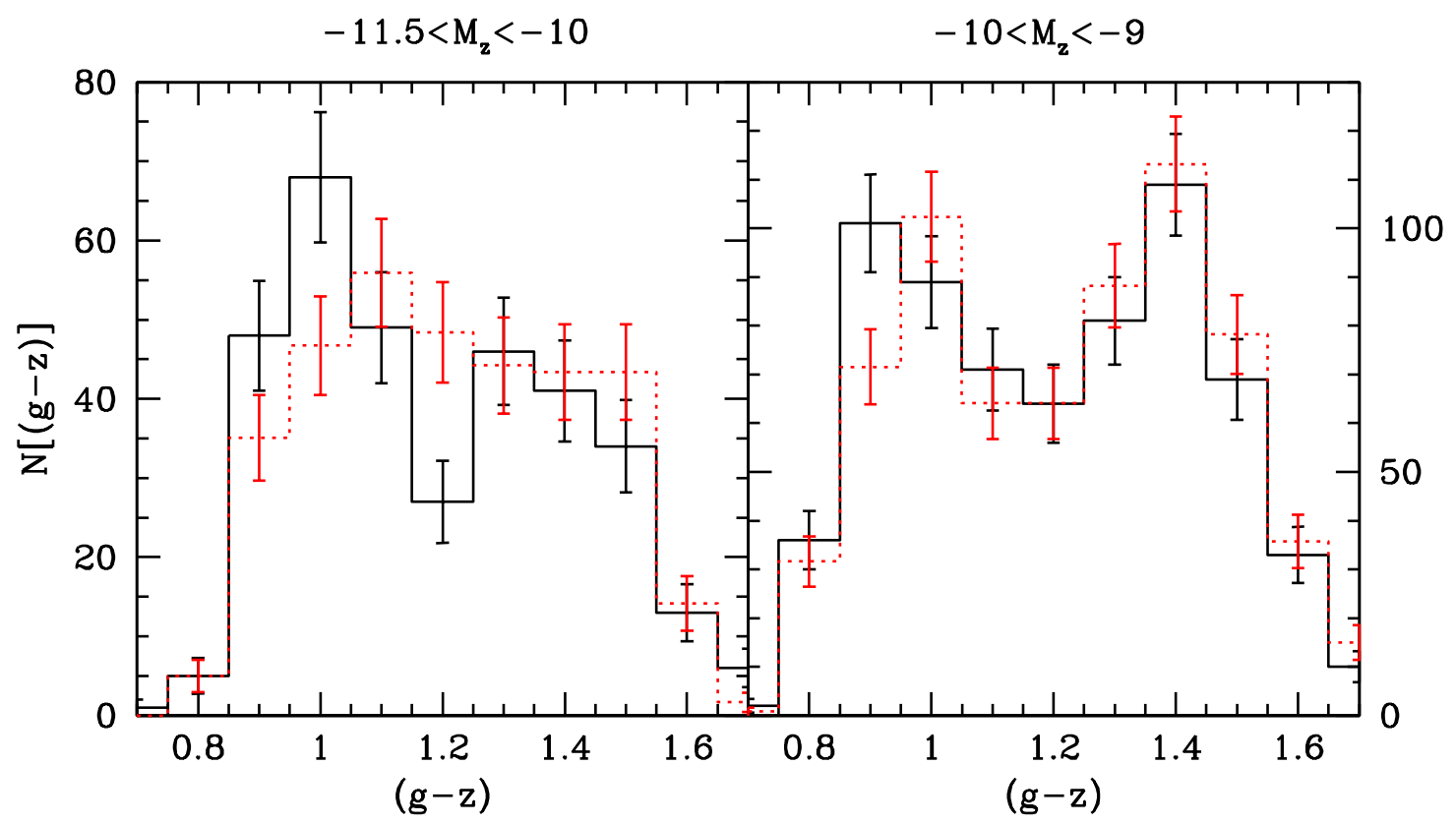

FIG. 9.- (Left Panel) GC color distributions for the two samples shown in Figure 8 The dotted histogram is the sample of inner GCs with $-11.5<M_{z}<-10 \mathrm{mag}$; the solid histogram is the sample of outer GCs with $-11.5<M_{z}<-10$ mag. (Right Panel) Same as in left panel, except for GCs with $-10<M_{z}<-9$ mag. The KS probability for the inner and outer GC samples having the same underlying distribution is $1 \%$ in the left panel and $14 \%$ in the right one. To facilitate the comparison, the histograms for the inner GCs have been rescaled by a factor of $1.2 \times$ in both plots, so that inner and outer sample have equal sizes.

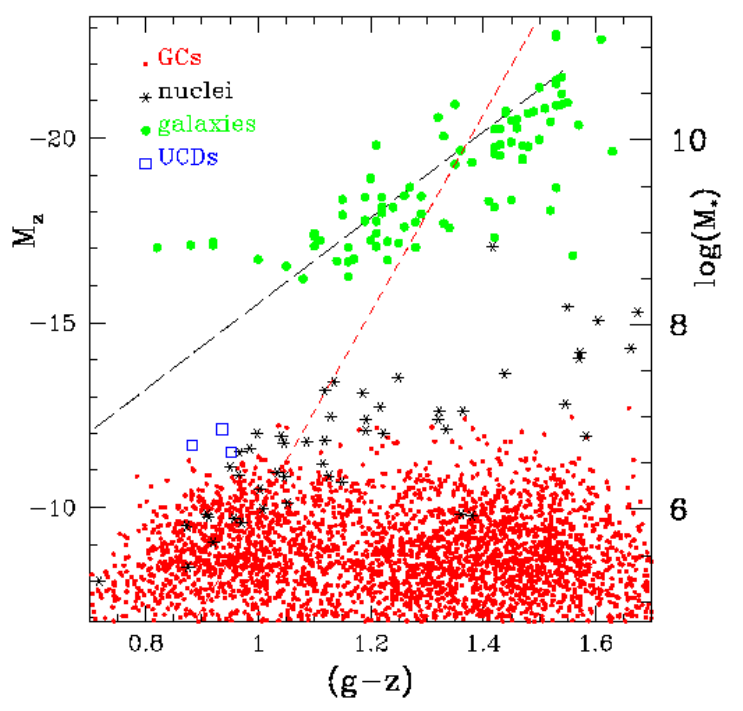

FIG. 10.- Color-magnitude diagram for GCs, compact stellar nuclei, early-type galaxies and UCDs in the Virgo Cluster. Small filled circles (online red) are GCs belonging to Group 1. The short dashed line is the KMM fit to the blue GC subpopulation, extrapolated to brighter luminosities. Asterisks are the nuclei of ACSVCS galaxies from Côté et al. (2006). Open squares (online blue) are the three UCD candidates from Haşegan et al. (2005) with ACS imaging. Large filled circles (online green) are the ACSVCS galaxies themselves (Ferrarese et al. 2006). The long dashed line indicates the $(V-I)$ color-magnitude relation for early-type galaxies in the Fornax Cluster from Hilker et al. (2003). The Fornax relation was transformed into $(g-z)$ according to $(g-z)=2.64(V-I)-1.72$, following Fukugita et al. (1996). The ordinate on the right denotes stellar masses, converted from $M_{z}$ using the mean $\mathrm{M}_{*} / \mathrm{L}_{z}$ ratio at metallicities of $[\mathrm{Fe} / \mathrm{H}]=-2.25$ and +0.56 dex for a $10-\mathrm{Gyr}$ population (Bruzual \& Charlot 2003), assuming a Chabrier (2003) IMF. Due to the low sensitivity of $M_{z}$ on metallicity, $\mathrm{M}_{*} / \mathrm{L}_{z}$ varies by just $40 \%$ between the two extreme metallicities, justifying a color independent luminosity-mass transformation for illustrative purposes.

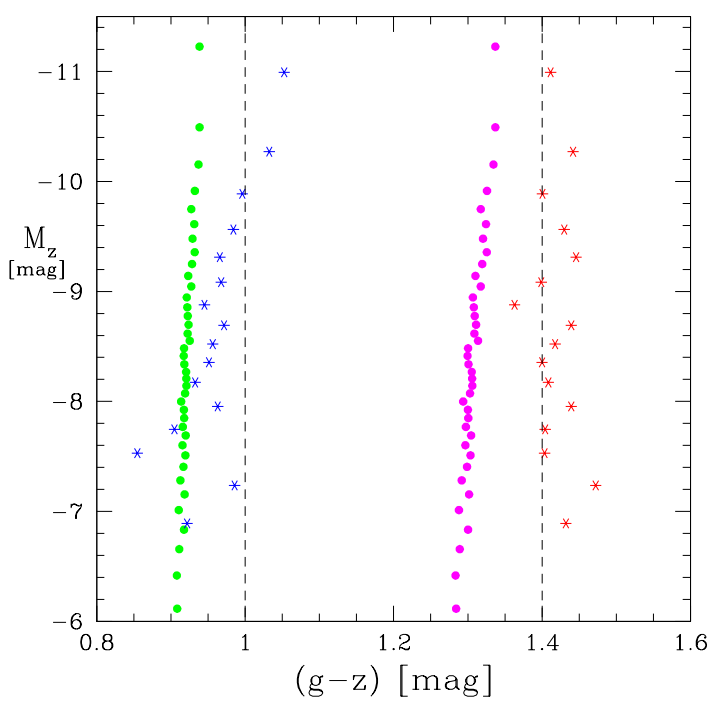

FIG. 11. - One test of the accretion scenario, showing a CMD in $M_{z}$ and $(g-z)$. Asterisks indicate the KMM fits to the blue and red subpopulations for GCs in Group 1 (see Figure 2). Dots show the mean GC color as expected from the mean host galaxy luminosity in each luminosity bin for the co-added CMDs of Groups 2-4. The tilt seen for the dots is the result of two effects: (1) the larger width of the GC luminosity function at brighter host galaxy magnitudes; and (2) the correlation between the mean GC color and host galaxy luminosity. See text for details.

al. 1998, Hilker et al. 1999a, Drinkwater et al. 2003, Haşegan et al. 2005, Kissler-Patig et al. 2006, Mieske et al. 2006). Since these two formation channels correspond to the two kinds of contaminators discussed in this Section, we conclude that also UCDs are unlikely to account for the observed trends.

\subsection{Accretion from Low-Mass Galaxies}


A population of GCs accreted from galaxies with a range of masses (e.g. Côté et al. 1998 or Hilker et al. 1999b) will exhibit a color-magnitude relation if: (1) the mean GC color of a single galaxy scales with galaxy mass; and (2) more massive GCs preferentially form in more massive galaxies. Indeed, both conditions are satisfied in actual early-type galaxies (see, e.g., Peng et al. 2005; Kundu \& Whitmore 2001; Jordán et al. 2006 in prep. and references therein). Moreover, this simple scenario may offer, at least in principal, a way to qualitatively understand the slope differences for individual galaxies (e.g., the non-existence of a correlation for M49) as a consequence of different accretion/merger histories.

In order to quantify the tilt in the CMD created by the accretion of GCs from low-mass galaxies that do not themselves have color-magnitude relations, we consider the combined GC sample of Groups 2-4 (see Figure 11). We first calculate the mean host galaxy magnitude for each bin, and then calculate the color in each GC bin according to the relation between host galaxy magnitude and mean color of the blue and red GC subpopulations (Table 3 of Peng et al. 2006). The outcome of this exercise is shown in Figure 11] As expected, there is a (weak) trend between mean color with luminosity for the GC subpopulations.

This trend is compared to the KMM fits for Group 1. There is a substantial offset in the sense that the fainter luminosity groups have bluer GC colors than Group 1. In addition, the simulated slope is substantially smaller than measured for the ensemble of galaxies in Group 1. All in all, we conclude that the observed color-magnitude relations are unlikely to have been created by accretion of GCs from low-mass galaxies, although we cannot rule out the possibility that accretion has contributed to the observed trend.

\subsection{Stochastic effects}

The light of old GCs is in the red optical to nearinfrared pass-bands dominated by red giant stars. Generally, the number of red giants is only a small fraction of the total number of stars in a given stellar population. It is therefore worth discussing to which extent stochastic effects due to small numbers of red giants can skew the probability distribution of the integrated GC luminosity. This may cause a trend of GC color with integrated luminosity, because any stochastic effect is weaker for higher GC luminosity (larger total number of stars) and for bluer bands (lower average stellar luminosity).

In the works by Cerviño et al. (2002), and Cerviño \& Luridiana (2004; 2006), the effect of discrete isochrone sampling on integrated photometric properties is discussed in detail. In Cerviño \& Luridiana (2006), the width, skewness and kurtosis for the probability distribution of integrated luminosity of a stellar population are calculated as a function of star number and age. The faint magnitude limit of our GCs $\left(M_{z} \simeq-7.7 \mathrm{mag}\right)$ corresponds to about $10^{5} M_{\odot}$, and hence about $2 \times 10^{5}$ stars. In the $I$-band - taken to represent the $z$ band due to lack of estimates for the latter band -, the photometric uncertainty arising from sampling effects amounts to about 0.06 mag for such a population (Cerviño \& Luridiana (2006); and Cerviño, private communications). The skewness of the distribution in integrated luminosity is about 0.25 . The product of these two numbers - about
$0.015 \mathrm{mag}$ - is a measure of the difference between mode and mean of the probability distribution in integrated luminosity. Given that the colour-magnitude trend in our data requires an almost ten times larger color shift, stochastic effects do probably not have a significant contribution to the observed trend.

There is also a phenomenological counterargument against the importance of stochastic effects: the colormagnitude trend is shaped more by brighter GCs (see Figure 9), while stochastic effects are stronger for fainter GCs. Furthermore, the environmental dependencies of the trend cannot be explained by purely intrinsic stochastic effects.

\subsection{Capture of Field Stars}

Given that the strength of the color-magnitude trend depends on local environment, it is reasonable to examine the extent to which capture of field stars may help to explain the observed slopes. The capture of field stars has previously been considered by Bica et al. (1997) for the case of a $10^{5} \mathrm{M}_{\odot}$ star cluster orbiting within the Galactic bulge at a distance of $1 \mathrm{kpc}$. They found that such a cluster could absorb a substantial fraction of its total mass during its lifetime. Similarly, Kroupa (1998) has shown that stellar super-clusters created in disks like that of the Milky Way can capture several tens of percent of their total mass in form of disk stars.

Field stars of the brightest early-type galaxies are significantly redder than their blue GC population, typically by about 0.5 to $0.6 \mathrm{mag}$ (Peng et al. 2006). Therefore, there will be a color shift towards the red by about $0.10-0.15$ for a blue GC by the time that $\approx 20-25 \%$ of the GC light originates from field stars. This effect will be notable mainly for the blue GC population, since the color of red GCs is much closer to that of field stars. As will become clear from the subsequent discussion, the capture rate per unit GC mass is higher for more massive GCs, which will lead to a slope in color-magnitude space. This qualitatively explains finding (1) from above. In addition, the effect will be stronger in the inner part of the host galaxy because of the higher field star density in such regions, consistent with finding (2). Thus, field star capture seems qualitatively consistent with the observed trend.

We now quantify this scenario by considering a GC with a mass of $10^{6} M_{\odot}$, a mass that roughly corresponds to the brightest clusters in Groups 1 and 2 in Figure 2 For an assumed $\mathrm{M} / \mathrm{L}=2.5$, this corresponds to a total of $4 \times 10^{5}$ solar luminosities. We assume the GC to be on a circular orbit around M87. This is certainly an extreme assumption, given that Côté et al. (2001) rule out such tangentially biased orbits for the GC system as a whole. We nevertheless use it for the sake of this simple estimate. We adopt a radius of $3 \mathrm{kpc}$, representative for the sample within $5 \mathrm{kpc}$ projected galactocentric distance.

We may estimate the number of captured stars by multiplying the total number of stars within the volume of influence of the GC with the fraction of stars having relative velocities below the GC escape velocity. As the volume of influence, we define a torus around the orbit of the GC with an inner radius of the GC's tidal radius with respect to its host galaxy M87. For calculating the tidal radius, we use the mass profile of M87 derived by Romanowsky \& Kochanek (2001). They quote the 
integrated mass at a distance of $3 \mathrm{kpc}$ to be $1.1 \times 10^{11}$ $M_{\odot}$, which is comparable to the value found by Côté et al. (2001). Together with the $10^{6} M_{\odot}$ mass of the $\mathrm{GC}$, this gives a tidal radius of $9 \mathrm{pc}$. The total volume of the torus then is $4.8 \times 10^{6} \mathrm{pc}^{3}$. We use the same paper by Romanowsky \& Kochanek to get the 3-dimensional stellar density of M87 at $3 \mathrm{kpc}$, which is about $0.06 \mathrm{~L}_{\odot}$ $\mathrm{pc}^{-3}$. That is, along one orbit there will be a total of 2.9 $\times 10^{5}$ solar luminosities within the volume of influence of our test GC.

However, only a small fraction of those will be captured: the average escape velocity $v_{e s c}$ of the GC within its tidal radius of $9 \mathrm{pc}$ is about $37 \mathrm{~km} \mathrm{~s}^{-1}$, while the stellar velocity dispersion in M87 is about $275 \mathrm{~km} \mathrm{~s}^{-1}$ (Romanowsky \& Kochanek 2001). The GC population in M87 has a velocity dispersion of $\sim 400 \mathrm{~km} \mathrm{~s}^{-1}$. The probability distribution for the relative velocity between the field stars and a GC is given by the convolution of both distributions, which is a Gaussian of width $\sigma=485$ $\mathrm{km} \mathrm{s}^{-1}$. The fraction of stars (in solar luminosities) that can be captured in the $1 \mathrm{D}$ case is given by the area below the Gaussian between $+/-\frac{v_{e s c}}{\sqrt{3}}$, which is 0.035 . For the full $3 \mathrm{D}$ case, this is $0.035^{3}=4.3 \times 10^{-5}$, or one out of $2.3 \times 10^{4}$ stars. Given that in total $2.9 \times 10^{5}$ stars are in the volume of influence in each orbit, this implies a capture rate of one dozen stars per orbit ${ }^{11}$. For a Keplerian orbit at $3 \mathrm{kpc}$, one revolution lasts about $2.5 \times 10^{7}$ years. In a Hubble time, one can therefore capture about 6000 stars. This is $1.5 \%$ of the total assumed GC luminosity, not sufficient to fully explain the observed effects. Only when considering a more extreme orbital distance of 1 kpc does the total number of captured stars increase to about $4 \times 10^{4}$, i.e. $\simeq 10 \%$ of all stars.

We now briefly examine the opposite effect of field star capture: namely cluster star evaporation. As is well known, mass segregation in GCs (Gunn \& Griffin 1979; Jordán 2004) will result in evaporation affecting mainly the lowest mass stars. Lamers et al. (2006) find for clusters of solar metallicity that evaporation will cause the overall GC color to become bluer with time (over most of their lifetime) due to the preferential loss of red, lowmass stars. Could such a color change help to explain our observations? Two effects are important: (1) the maximum blueward shift due to evaporation is larger for more massive clusters (Lamers et al. (2006)), reaching up to $\Delta(V-I) \simeq 0.10 \mathrm{mag}$; and (2) the dissolution time, $t_{\text {diss }}$, is longer for more massive clusters. To qualitatively explain our observations one would require the massive GCs to be in an early stage of evolution, so that evaporation has not yet had a significant effect. The fainter GCs would mainly define the color magnitude trend. However, Figure 9 shows that the slope changes between inner and outer GCs are mainly driven by the brightest GCs. That is, evaporation is unlikely to account fully for the observed color-magnitude trend. Even more serious, evaporation cannot naturally explain the simultaneous presence of a trend for the blue GCs and its absence for the red.

\footnotetext{
11 Note that close encounters with GC stars may indeed alter the capture rate, but an accurate assessment of this effect would require dedicated $N$-body simulations, which is beyond the scope of this paper.
}

\subsection{GC Self Enrichment}

Generally speaking, self enrichment will occur in any stellar system, GCs included, if the system is able to retain a fraction of the gas expelled by the first generation of supernovae and subsequently form a new generation of stars. To create a metallicity-mass (or, equivalently, a color-magnitude) trend via self-enrichment, one would therefore require that more massive GCs are more efficiently self-enriched. The extent to which GCs in the Milky Way may have self-enriched, however, is a controversial subject: e.g., Frank \& Gisler (1976), Smith (1996), Gnedin et al. (2002), Parmentier \& Gilmore (2001), Dopita \& Smith (1986), Morgan \& Lake (1989), Thoul et al. (2002).

In this context, it is interesting to note that the most massive GC in the Galaxy, $\omega$ Cen, is known to have a metallicity distribution function that shows a long tail towards high metallicities (e.g., Norris, Freeman \& Mighell 1996; Stanford et al. 2006). Various studies have found this high-metallicity population to be either coeval, or perhaps 2-4 Gyr younger, than the cluster's primary (metal-poor) population (e.g., Ferraro et al. 2004; Sollima et al. 2005; Hughes \& Wallenstein 2000; Hilker \& Richtler 2000; Rey et al. 2004, Stanford et al. 2006). This is consistent with $\omega$ Cen having been at least partially self-enriched. Note, however, that because of its peculiar star formation history, numerous investigators have suggested that the cluster may be the stripped nucleus of a now-dissolved dwarf galaxy (e.g., Freeman 1993; Hilker \& Richtler 2000; Bekki \& Freeman 2003). If true, then it may be incorrect to consider $\omega$ Cen to be a typical example of a self enriched GC.

This caveat aside, if self enrichment is common to the formation of the most massive GCs, then it may indeed help to explain our observations. Most significantly, the lack of a correlation among the red GC subpopulation is naturally explained in this scenario: i.e., the redder GCs are already metal-enriched, and a fixed amount $d \mathrm{Z}$ of supernovae ejecta will cause a relatively weaker metallicity increase, $d[\mathrm{Fe} / \mathrm{H}]$, for metal-rich GCs. For instance, for a system with $[\mathrm{Fe} / \mathrm{H}]=-2(\mathrm{Z}=0.0002)$, adding a fixed amount of metals $d \mathrm{Z}=0.0002$ will result in $d[\mathrm{Fe} / \mathrm{H}]=+0.3$ dex. According to Peng et al. (2006) this corresponds to a shift in $(g-z)$ of about 0.06 mag and is about the observed amount of the color magnitude trend. However, for a system with $[\mathrm{Fe} / \mathrm{H}]=-0.5$ and the same $d \mathrm{Z}$, we only have $d[\mathrm{Fe} / \mathrm{H}]=+0.013 \mathrm{dex}$, with a corresponding color change in $(g-z)$ well below 0.01 mag.

A possible shortcoming of this self-enrichment scenario is that is does not explicitly account for environmental effects such as a dependence on galactocentric radius. On the other hand, feedback induced by pressureconfinement from the surrounding medium may be able to help reconcile this scenario with observations.

\subsection{The Distribution of GCs in Metallicity-Mass Space}

To conclude this discussion, we show in Figure 12 a metallicity-luminosity plot for the co-added sample of GCs in Group 1. To estimate GC metallicities, we have applied a quartic color-metallicity transformation based on the empirical calibration data from Peng et al. (2006). Note that the resulting metallicity distribution is not bi- 


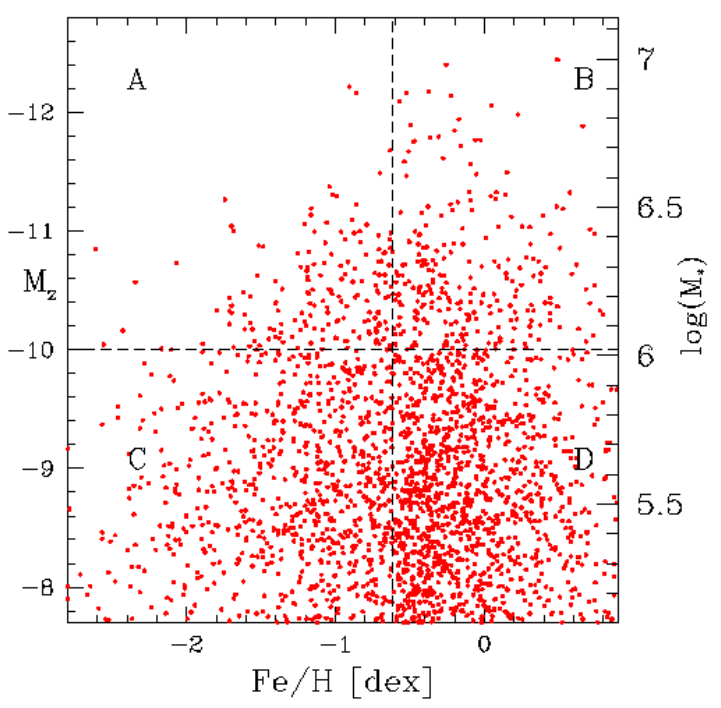

FIG. 12.- Metallicity plotted as a function of stellar mass and $M_{z}$ for GCs in Group 1, assuming a quartic color-metallicity transformation based on the empirical calibration data of Peng et al. (2006). Conversion from luminosity to stellar mass is performed as in Figure 10 The vertical dashed line corresponds to the limiting color between blue and red GC subpopulations from Figure 5] The horizontal dashed line corresponds to the dividing point between brighter and fainter GCs from Figure 9 A KS test shows that the metallicity distributions in quadrant A and $\mathrm{C}$ stem from the same underlying distribution with only $0.1 \%$ probability. This probability is $91 \%$ for the color distributions in quadrants B and D. The probability for the joint sample of quadrants A and B compared to the joint samples $\mathrm{C}$ and $\mathrm{D}$ is $16 \%$.

modal, but rather shows a single pronounced peak at $[\mathrm{Fe} / \mathrm{H}] \simeq-0.4$ dex and a long, extended tail towards lower metallicities. This confirms recent warnings (e.g., Richtler 2005; Yoon, Yi \& Lee 2006) that bimodality in color does not necessarily imply bimodality in metallicity.

Using the data in Figure 12 we can compare the metallicity distributions of GCs brighter and fainter than $M_{z}=-10$ mag. For the metal-poor population, $([\mathrm{Fe} / \mathrm{H}]<-0.6)$, the fainter GCs have a significantly broader tail towards lower metallicities than do the bright ones. A KS-test gives a $0.1 \%$ probability for a common underlying distribution between both samples. In the metal-rich part of the diagram, the agreement is very good, with a KS probability of $91 \%$. In other words, the empirical finding of a color-magnitude trend for the blue GCs can hence be re-phrased in terms of metallicity: the GC systems appear to have a skewed, but unimodal, metallicity distribution function whose metalpoor tail becomes increasingly extended at lower luminosities/masses.

\section{SUMMARY AND CONCLUSIONS}

In this paper we have analysed the color-magnitude relation for the GC subpopulations in early-type galaxies belonging to the Virgo Cluster. Our sample consists of 79 galaxies, spanning the range $-21.7<M_{B}<$ $-15.2 \mathrm{mag}$, drawn from the ACS Virgo Cluster Survey (Côté et al. 2004). Our principal findings can be summarized as follows:

1. Based on KMM fits, we find a highly significant correlation $\gamma_{z} \equiv \frac{d(g-z)}{d z}=-0.037 \pm 0.004$ for the blue
GCs in the co-added CMD of the three brightest Virgo Cluster galaxies (M49, M87, M60). The sense of the correlation is such that brightest GCs in this subpopulation are redder than their faint counterparts. For M87 and M60 alone, we find comparable correlations; by contrast, the blue GCs in M49 do not appear to follow such a trend. In no galaxy do we find a significant correlation between $(g-z)$ and $M_{z}$ for the GCs associated with the red subpopulation.

2. Based on KMM fits, we find the slope $\gamma_{g} \equiv \frac{d(g-z)}{d g}$ within the blue subpopulation to be much weaker than $\gamma_{z}$. In turn, we find a mild positive correlation $\gamma_{g}$ for the red subpopulation, in the opposite sense to what is found for the blue subpopulation.

3. We test KMM on artificial CMDs with, and without, implemented slopes for the blue subpopulation, and no slope for the red population. KMM correctly reproduces the implemented slopes when using the CMD $(g-z)$ vs. $M_{z}$. For $(g-z)$ vs. $M_{g}$, KMM gives a biased estimate for the color of the blue population and yields a positive slope for the red subpopulation, although no slope had been implemented. We trace this bias to the fact that in $M_{g}-$ unlike in $M_{z}$ - the luminosity function of blue GCs is populated to significantly brighter luminosities than for the red GCs. We conclude that for the real data, the strong slope $\gamma_{z}$ is not an artifact of the fitting method, while the weaker slope $\gamma_{g}$ is an artificial feature of the KMM fit.

4. We derive correlations independent of KMM by using a biweight estimator for GCs divided on the basis of a luminosity independent color. The results confirm the significant slope found by KMM in $M_{z}$ and reveal no significant discrepancies between the slope measured using $M_{z}$ and $M_{g}$.

5. The slope measured from the blue subpopulation decreases with host galaxy luminosity. However, even among the faintest galaxies $\left(-18.4<M_{B}<\right.$ $-15.2 \mathrm{mag})$ the slope is nonzero.

6. The color-magnitude relation exhibited by the blue GCs is stronger for clusters with smaller projected galactocentric distances. This difference is driven mainly by GCs with $M_{z}<-10 \mathrm{mag}$.

We examine a number of physical mechanisms that might give rise to a color-magnitude relation with these characteristics: (1) contamination by super-star-clusters, stripped galactic nuclei, or ultra-compact dwarf galaxies; (2) accretion of GCs from low-mass galaxies; (3) stochastic effects; (4) capture of galactic field stars by GCs; and (5) self-enrichment of individual GCs. Neither contaminants nor accretion of low-mass galaxies appear likely to fully explain our observations. Self-enrichment in more massive GCs may be able to account for the observed color-magnitude relation, although more work is needed to determine if the dependence on galactocentric radius can be explained within the context of the selfenrichment scenario. Although the capture of field stars does lead to a dependence on galactocentric radius that 
is at least qualitatively in agreement with our observations, this mechanism does not appear efficient enough to explain the overall size of the observed trend.

While none of these scenarios can fully account for the full set of observations in a straightforward manner, self-enrichment and capture of field stars seem the most promising approaches. There is, of course, no a priori reason that a single mechanism is responsible for the observed color-magnitude relation, and conceptually, the combined effects of self-enrichment and field star capture may be able to explain the observations: i.e., field star capture could act as a second order effect, producing environmental variations of a global mass-metallicity relation induced by self-enrichment. This would alleviate the discrepancy between real and required field star capture rate estimated in this work. Dedicated N-body simulations going beyond these simple estimates will be required to accurately address the efficiency of field star capture in different environments.

We would like to thank Pavel Kroupa, Mike Fellhauer, Holger Baumgardt, H.J.G.L.M. Lamers and Miguel
Cerviño for fruitful discussions. We thank the anonymous referee for her/his comments. Support for program GO-9401 was provided through a grant from the Space Telescope Science Institute, which is operated by the Association of Universities for Research in Astronomy, Inc., under NASA contract NAS5-26555. P.C. acknowledges support provided by NASA LTSA grant NAG511714. M.J.W . acknowledges support through NSF grant AST-0205960. S.M. acknowledges additional support from NASA grant NAG5-7697 to the ACS Team. D.M. acknowledges support provided by NSF grants AST0071099, AST-0206031, AST-0420920 and AST-0437519, by NASA grant NNG04GJ48G, and by grant HST-AR09519.01-A from STScI. This research has made use of the NASA/IPAC Extragalactic Database (NED) which is operated by the Jet Propulsion Laboratory, California Institute of Technology, under contract with the National Aeronautics and Space Administration.

\section{REFERENCES}

Ashman, K. M., Zepf, S. E. 1992, ApJ, 384, 50

Ashman, K. M., Bird, C. M., Zepf, S. E. 1994, AJ, 108, 2348

Bastian, N., Emsellem, E., Kissler-Patig, M., Maraston, C. 2006, A\&A, 445, 471

Bassino, L. P., Muzzio, J. C., \& Rabolli, M. 1994, ApJ, 431, 634

Beasley, M. A. et al. 2002, MNRAS, 333, 383

Beers, T. C., Flynn, K., Gebhardt, K. 1990, AJ, 100, 32

Bekki, K., Couch, W.J., Drinkwater, M.J., Shioya, Y., 2003a, MNRAS, 344, 399

Bekki, K., \& Freeman, K. C. 2003, MNRAS, 346, L11

Bekki, K., Couch, W.J., Drinkwater, M.J., Shioya, Y., 2004, ApJL, 610, 13

Bica, E. et al. 1997, ApJ, 482, 49

Bower, R.G., Lucey, J.R., Ellis, R.S. 1992, MNRAS, 254, 589

Bruzual, G., Charlot, S. 2003, MNRAS, 344, 1000

Burgarella, D., Kissler-Patig, M., Buat, V. 2001, AJ, 121, 2647

Cerviño, M., Valls-Gabaud, D., Luridiana, V., \& Mas-Hesse, J. M. 2002, A\&A, 381, 51

Cerviño, M., Luridiana, V. 2004, A\&A, 413, 145

Cerviño, M., Luridiana, V. 2006, A\&A, 451, 475

Chabrier, G., 2003, PASP, 115, 763

Côté, P., Marzke, R. O., West, M. J. 1998, ApJ, 501, 554

Côté, P. et al. 2001, ApJ, 559, 828

Côté, P., McLaughlin, D. E., Cohen, J. G., \& Blakeslee, J. P. 2003, ApJ, 591, 850

Côté, P. et al. 2004, ApJS, 153, 223 (Paper I)

Côté, P. et al. 2006, ApJS, accepted, astro-ph/0603252 (Paper VIII)

Dirsch, B. et al. 2003, AJ, 125, 1908

Dopita, M. A., Smith, G. H. 1986, ApJ, 304, 283

Drinkwater, M.J., Gregg, M.D., Hilker, M. et al., 2003, Nature, 423,519

Fellhauer, M., Kroupa, P., 2002, MNRAS, 330, 642

Fellhauer, M.; Kroupa, P., 2005, MNRAS, 359, 223

Ferraro, F.R. et al. 2004, ApJ, 603, L81

Ferrarese, L. et al. 2006, ApJS, 164, 334 (Paper VI)

Freeman, K. C. 1993, in ASP Conf. Ser. 48, The Globular ClusterGalaxy Connection, ed. G. H. Smith \& J. P. Brodie (San Francisco: ASP), 27

Forbes, D. A., Brodie, J. P., Grillmair, C. J. 1997, AJ, 113, 1652

Ford, H. C., Bartko, F., Bely, P. Y. et al. 1998, Proc. SPIE Vol 3356, p. 234-248, Space Telescopes and Instruments V, Pierre Y. Bely; James B. Breckinridge; Eds.

Frank, J., Gisler, G. 1976, MNRAS, 176, 533

Fukugita, M., Ichikawa, T., Gunn, J.E. et al. 1996, AJ, 111, 1748

Gebhardt, K., Kissler-Patig, M. 1999, AJ, 118, 1526

Gnedin, O. Y. et al. 2002, ApJ, 568, 23

Gunn, J. E., Griffin, R. F. 1979, AJ, 84, 752
Harris, G. L. H., Harris, W. E., Geisler, D. 2004, AJ, 128, 723

Harris, W. E. et al. 2006, ApJ, 636, 90

Hasegan, M., Jordán, A., Côté, P. et al. 2005, ApJ, 627, 203

Hilker, M., Infante, L., Vieira, G., Kissler-Patig, M., Richtler, T. 1999, A\&AS, 134, 75

Hilker, M., Infante, L., Richtler, T. 1999, A\&AS, 138, 55

Hilker, M., \& Richtler, T. 2000, A\&A, 362, 895

Hilker, M., Mieske, S., \& Infante, L. 2003, A\&AL, 397, L9

Hughes, J., \& Wallerstein, G. 2000, AJ, 119, 1225

Jones, J. B., et al. 2006, AJ, 131, 312

Jordán, A., Blakeslee, J. P., Peng, E. W. et al. 2004a, ApJS, 154, 509 (Paper II)

Jordán, A., Côté, P., Ferrarese, L. et al. 2004b, ApJ, 613, 279 (Paper III)

Jordán, A. 2004, ApJ, 613, L117

Jordán, A., Côté, P., Blakeslee, J. et al. 2005, ApJ, 634, 1002 (Paper $\mathrm{X})$

Karick, A., Drinkwater, M.J., Gregg, M.D. 2003, MNRAS, 344, 188 Kissler-Patig, M. 1997, A\&A, 319, 83

Kissler-Patig, M., Jordán, A., Bastian, N. 2006, A\&A, 448, 1031

Kravtsov, A. V., Gnedin, O. Y. 2005, ApJ, 623, 650

Kroupa, P. 1998, MNRAS, 300, 200

Kundu, A., Whitmore, B. 2001, AJ, 121, 2950

Lamers, H.J.G.L.M, Anders, P., de Grijs, R. 2006, A\&A, 452, 131

Larsen, S. S., Brodie, J. P., Huchra, J. P., Forbes, D. A., Grillmair, C. J 2001, AJ, 121, 2974

Lotz, J. M., Miller, B. W., Ferguson, H. C. 2004, ApJ, 613, 262

Mei, S. et al. 2005, ApJ, 625, 121 (Paper V)

Mei, S. et al. 2006, submitted to ApJ (Paper XIII)

Mieske, S., Hilker, M., Infante, L. \& Jordán, A. 2006, AJ 131, 2442

Minniti, D., Kissler-Patig, M., Goudfrooij, P., Meylan, G. 1998, AJ, 115, 121

Morgan, S., Lake, G. 1989, ApJ, 339, 171

Norris, J. E., Freeman, K.C., Mighell, K.J. 1996, ApJ, 462, 241

Odenkirchen, M., Grebel, E. K., Dehnen, W. 2003, AJ, 126, 2385

Ostrov, P. G., Forte, J. C., Geisler, D. 1998, AJ, 116, 2854

Parmentier, G., Gilmore, G. 2001, A\&A, 378, 97

Peng, E., et al. 2006, ApJ, 639, 95 (Paper IX)

Pryor, C., McClure, R. D., Fletcher, J. M., Hesser, J. E. 1989, AJ, 98, 596

Rey, S. C. et al. 2004, AJ, 127, 958

Richardson, S., \& Green, P. G. 1997, JR. Statist. Soc. B, 1997, 59, 731

Richtler, T. 2005, in press at Bull. Astron. Soc. India, astro-ph/0512545

Romanowsky, A., \& Kochanek, C.S. 2001, ApJ 553, 722

Schlegel, D.J., Finkbeiner, D.P., \& Davis, M. 1998, ApJ, 500, 525

Searle, L, Zinn, R. 1978, ApJ, 225, 357 
Smith, G.H. 1996, PASP, 108, 176

Sollima, A. et al. 2005, ApJ, 634, 332

Spitler, L. R., et al. 2006, AJ accepted, astro-ph/0606337

Stanford, L. M., da Costa, G. S., Norris, J. E. \& Cannon, R. D. 2006, ApJ, in press, astro-ph/0605612

Strader, J., Brodie, J.P., Spitler, L., Beasley, M.A. 2006, AJ submitted, astro-ph/0508001

Thoul, A. et al. 2002, A\&A, 383, 491
Tonry, J.L., Dressler, A., Blakeslee, J.P. et al. 2001, ApJ, 546, 681 West, M. J., Côté, P., Marzke, R. O., Jordán, A. 2004, Nature, 427, 31

Whitmore, B. C. et al. 1995, ApJ, 454L, 73

Yoon, S., Yi, S. K., Lee, Y. 2006, Science, 311, 1129 